\title{
Hémorhéologie et écoulements diphasiques dans le système vasculaire
}

\author{
Haemorheology and two-phase flow in the vascular system
}

\author{
Daniel Quemada \\ Laboratoire de Biorhéologie, Université Paris VII \\ Equipe de Recherche associée au CNRS
}

\section{Propriétés générales des écoulements sanguins}

\section{Introduction}

Le sang, ainsi que la grande majorité des fluides biologiques, contient des éléments figurés. On ne peut donc plus le considérer comme un fluide homogène dès que l'échelle $R$ du conduit dans lequel il s'écoule n'est plus très grande devant la dimension caractéristique $a$ de ces éléments. Le caractère diphasique des écoulements sanguins résulte de cette inhomogénéité et apparait alors comme une des principales manifestations de l'existence d'une relation comportement-structure pour le sang.

Du point de vue de l'hydrodynamicien, l'hydrodynamique sanguine pose un certain nombre de problèmes spécifiques, rencontrés principalement:

1. dans la nature du sang,

2. dans les caractéristiques des vaisseaux (forme, structure et propriétés rhéologiques ; singularités géométriques, normales ou pathologiques),

3. dans la nature des écoulements, pulsés ou intermittents, quelquefois instables, voire turbulents,

4. dans l'importance des phénomènes de transport de masse (au sein du fluide ainsi qu'au travers des parois vasculaires) et de leur rétroaction sur les propriétés mécaniques du sang et des parois,

5. dans l'intervention, in vivo, de mécanismes complexes de contrôle et de régulation (pression et rythme cardiaque, diamètre des vaisseaux, composition $\mathrm{du}$ sang...).

Ces différentes caractéristiques se retrouvent, à des degrés divers, dans les trois grands domaines, eux-mêmes très vastes, qu'il est possible de distinguer en hydrodynamique sanguine : la grande (et la petite) circulation, la microcirculation et la circulation capillaire, cette der- nière étant habituellement incluse dans la microcirculation. Mais on peut retenir,pour l'essentiel, que ce sont:

(i) les propriétés rhéologiques des vaisseaux (artères et veines principales) qui interviennent dans la grande circulation,

(ii) le caractère non-newtonien du sang qui domine dans la microcirculation,

(iii) les propriétés mécaniques des globules rouges, notamment celles de leurs membranes, qui commandent la circulation capillaire (siège des échanges transpariétaux, donc associée aussi à la perméabilité des parois capillaires).

Le présent exposé va tenter de dégager le plus simplement possible, les caractères généraux de ces deux derniers domaines, où le sang ne peut plus être considéré comme homogène. Il laissera de côté beaucoup de questions dont l'intérêt biologique ou médical est fondamental, notamment celles concernant la coagulation.

Composition du sang.

Propriétés du globule rouge et du plasma

Le sang est une suspension très concentrée (environ $45 \%$ en volume) d'éléments cellulaires (globules rouges et blancs, plaquettes. . ) ; le liquide suspendant est du plasma, solution aqueuse d'électrolytes et de substances organiques, en grande partie des protéines.

$\mathrm{La}$ concentration en volume des globules rouges est déterminée par centrifugation, qui permet de séparer les cellules du plasma. La fraction du volume occupée par les cellules est l'hématocrite $H$, qui ne donne qu'une valeur approchée de la concentration volumique vraie $c$. Lorsque cette séparation résulte du phénomène de coagulation, on obtient un caillot et du sérum. Le sérum est pratiquement du plasma privé d'une des protéines 
plasmatiques, le fibrinogène, qui est à l'origine la formation de fibrine constituant la trame du caillot.

Les proportions relatives des éléments figurés et des constituants plasmatiques sont les suivantes (Cf., par exemple Burton, 1966).

$\begin{array}{lr}\begin{array}{l}\text { Eléments figurés }\left(5 \times 10^{6} \text { particules } / \mathrm{mm}^{3}\right) \\ \text { Globules blancs (de toutes sortes) }\end{array} & 1 \\ \text { Plaquettes } & 30 \\ \text { Globules rouges } & 600 \\ & \text { (proportions relatives) } \\ \text { Plasma } & \% \\ \text { Eau } & 0,91 \\ \text { Eléments inorganiques } & 0,01 \\ \text { Protéines } & 0,07 \\ \text { Autres éléments organiques } & 0,01\end{array}$

La supériorité numérique des Globules Rouges entraîne, en première analyse, la possibilité de négliger, $\mathrm{du}$ point de vue mécanique, la présence des autres particules.

a) Les globules rouges sont des disques biconcaves dont les dimensions $(\simeq 8 \mu \mathrm{m}$ de diamètre $\simeq 2 \mu \mathrm{m}$ d'épaisseur) dépassent la taille critique associée au mouvement brownien. Le sang, au repos, montre donc une sédimentation (c'est d'ailleurs la vitesse de cette demière qui est utilisée en clinique ( $\nu_{\mathrm{s}} \simeq 1 \mathrm{~cm} /$ heure $)$ ). Néanmoins, la différence de masse spécifique étant très faible $\left(\rho \simeq 1,05 \mathrm{gr} / \mathrm{cm}^{3}\right)$ la mise en écoulement rend cette sédimentation négligeable et la suspension peut être considérée comme stable. Le contenu des globules rouges est constitué surtout d'hémoglobine, en solution saturée mais de structure inconnue (cristal liquide (?)), qui lui confère des propriétés différentes (notamment une viscosité plus faible, semble-t-il) de celle de la solution préparée in vitro, à même concentration $(35 \mathrm{mg}-\mathrm{Hb} / 100 \mathrm{ml}-$ plasma). La membrane du Globule Rouge normal possède une déformabilité extraordinaire (analogue à celle d'un ballon mince partiellement dégonflé, (Brochard, 1977)), tout au moins tant que le Globule Rouge est dans des conditions normales. Lorsque pour une pression osmotique inférieure à la normale le globule a pris une forme sphérique, la membrane a un comportement viscoélastique (Cf. Rand, 1964), et présente une grande fragilité (donnant lieu à l'hémolyse) lorsqu'elle est soumise à l'action de toute contrainte d'assez longue durée (contrainte d'origine osmotique ou, comme dans la plupart des appareils d'assistance circulatoire, d'origine mécanique (chocs contre les parois, grands cisaillements de vitesse)).

b) Le plasma se comporte comme un fluide newtonien (de viscosité $\eta_{p}$ ), tout au moins tant que les protéines plasmatiques n'ont pas été dénaturées (par action de la chaleur ou de l'air, par ex.). Celles-ci sont de quatre types : l'albumine et les globulines, qui en représentent la presque totalité, et les lipo-protéines et le fibrinogène. $\mathrm{Ce}$ dernier joue un rôle très important, non seulement dans le phénomène de coagulation (formation de fibrine), mais aussi, en conditions normales, et associé aux $\beta$ globulines, dans le phénomène d'agrégation reversible des Globules Rouges, sous forme de rouleaux. Cette agrégation reversible dépend de la vitesse de cisaillement et influera fortement sur les propriétés rhéologiques du sang.

\section{La circulation capillaire}

\section{Caractéristiques générales}

$\mathrm{La}$ fonction essentielle de la circulation capillaire est d'assurer le transport transpariétal $\left(0_{2}, \mathrm{CO}_{2}\right.$, matériaux nutritifs et déchets) : ces échanges transcapillaires sont donc de toute première importance du point de vue physiologique. Néanmoins, du point de vue hydrodynamique, le flux de fluide accompagnant ces échanges est très faible (le rapport flux radial-flux axial est de l'ordre de $10^{-3}$ dans des conditions normales) et, peut être négligé, sauf s'il en résultait d'éventuelles modifications du Globule Rouge (par variation de $\mathrm{pH}$, par ex.) venant perturber l'écoulement.

Cette microcirculation a lieu dans les capillaires approximativement cylindriques et rigides $(2 \leqslant R \leqslant$ $4 \mu \mathrm{m}$ ) analogues à des tunnels creusés dans un gel. Les Globules Rouges ne les traversent aisément (à une vitesse de l'ordre de $1 \mathrm{~mm} / \mathrm{sec}$ ) que grâce à leur extrême déformabilité. La circulation capillaire sera donc dominée par les propriétés mécaniques $d u G R$, superposition de celles de sa membrane et de celles du fluide contenu à l'intérieur de cette membrane.

La figure 1 montre les Globules Rouges se déplaçant en file unique, avec une forme en parachute, et un espacement plus ou moins régulier : c'est l'écoulement par embols ("bolus flow"). Le degré de déformation qui dépend à la fois du rayon $R$ du capillaire et du débit $Q$,

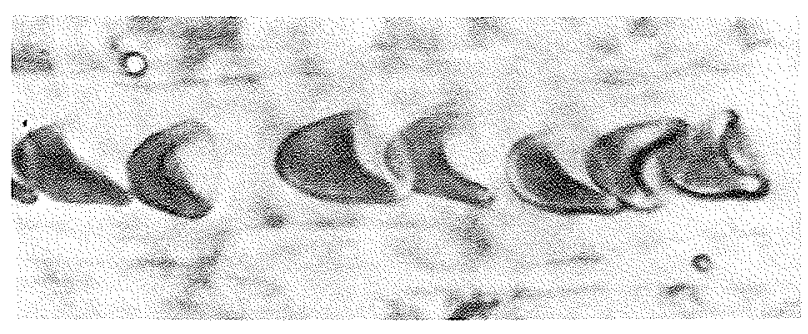

a)

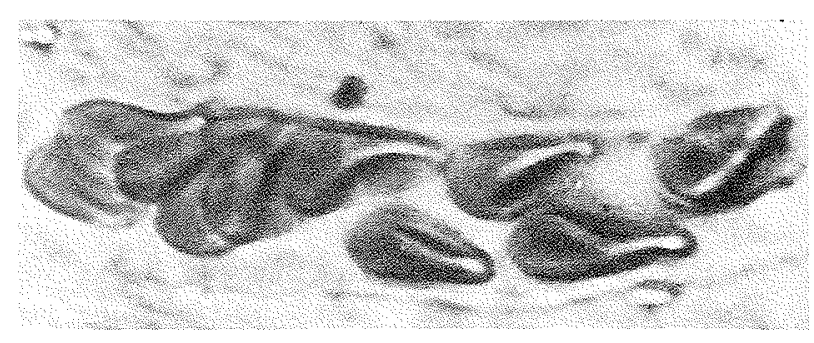

b)

Figure 1 - Déformation des Globules Rouges humains dans : a) un capillaire d'environ $7 \mu \mathrm{m}$ de diamètre

b) un vaisseau étroit de $12 \mu \mathrm{m}$ de diamètre (d'après Skalak et Branemark, 1969) 
est minimum pour $R$ grand et $Q$ faible. Cet écoulement diphasique s'apparente à celui de bulles immergées, traversant un tube étroit.

La présence du Globule Rouge doit avoir pour conséquences:

(i) l'augmentation de viscosité apparente

(ii) l'augmentation (éventuelle) du transport diffusif.

\section{a) Augmentation de la viscosité}

Un globule rouge perturbe le fluide dans son voisinage et cette perturbation augmente les pertes d'énergie par viscosité. Un très grand nombre de modèles ont été développés, d'abord pour une particule isolée, axisymétrique, puis pour plusieurs particules, régulièrement espacées. Nous rappellerons brièvement les principaux résultats.

Les mc dèles numériques ont porté soit sur les particules rigides [disques (Lew et Fung, 1970), sphères, particules de même forme que le Globule Rouge déformé ou non, ou que les rouleaux de Globules Rouges (Skalak et al., 1972)], soit sur les particules déformables (bulles sphériques ou bulles longues). Ils permettent d'évaluer la chute de pression supplémentaire, et partant la viscosité apparente, en fonction du rapport $a_{1} / R$, $a_{1}$ étant le "rayon" de la particule in situ, et de la distance $2 L=2 \lambda R$ séparant deux particules consécutives.

Les modèles hydrauliques ont utilisé des capsules de latex (emplies d'huile de silicone) dont les caractéristiques sont ajustées par analyse dimensionnelle. Nous nous limiterons ici aux résultats de Sutera et al., (1970). L'observation montre que, comme les Globules Rouges, les particules prennent approximativement une position axisymétrique, avec une couche annulaire de plasma, réduisant le diamètre $D_{c}=2 a$ des particules, au diamètre effectif $d_{c}=2 a_{1}<D_{t}=2 R$. Ce diamètre $d_{c}$ diminue lorsque la vitesse moyenne $U$ augmente, et tend vers une limite pour $U \geqslant 2 \mathrm{~mm} / \mathrm{sec}$. Simultanément, au fur et à mesure que la déformation de la particule augmente, sa vitesse $U_{c}$ augmente aussi et tend vers la limite $U_{c}=2 U$.

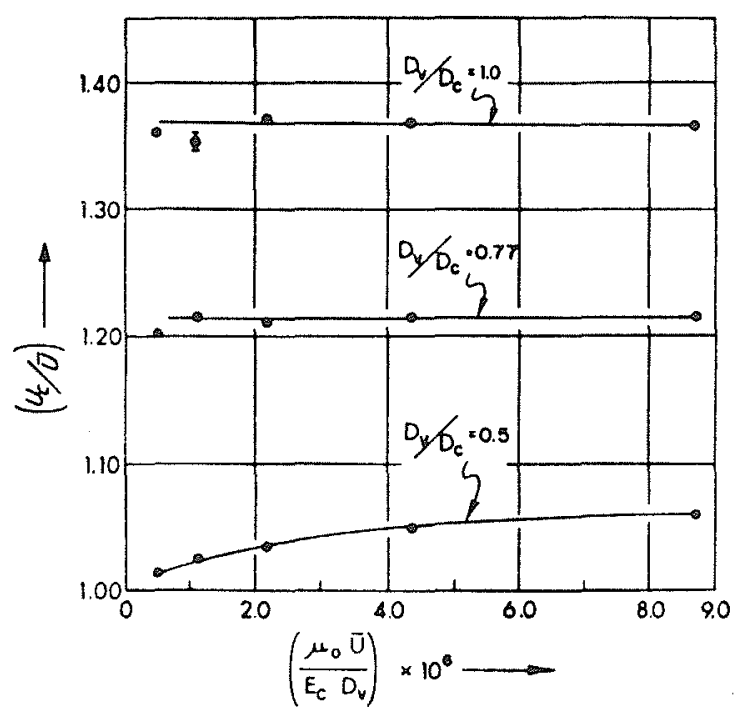

Figure 2 - Vitesse relative des Globules Rouges en fonction du paramètre de déformabilité $V$. (d'après Sutera et al., 1970).

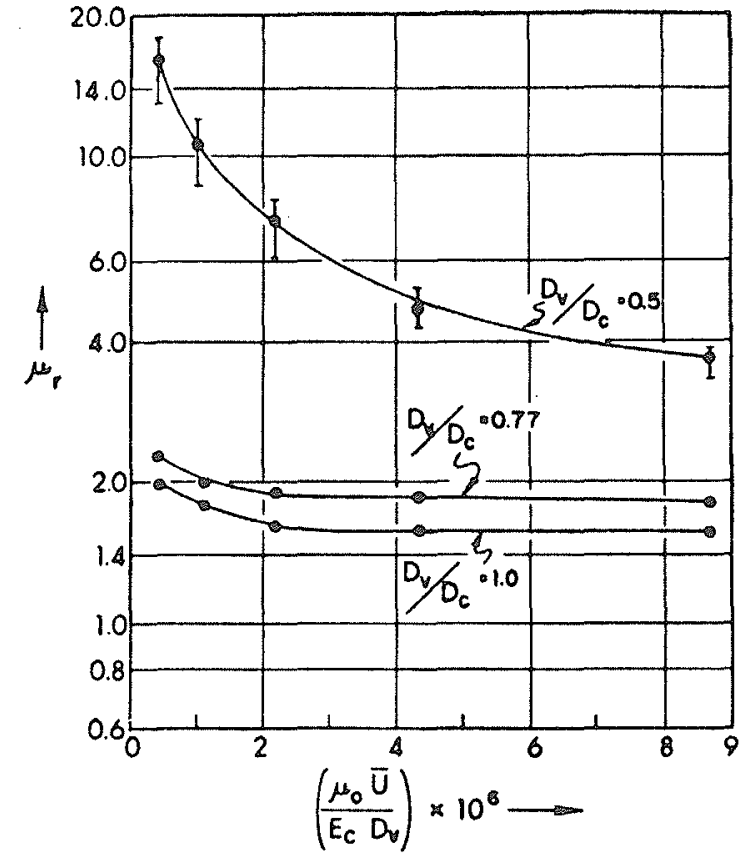

Figure 3 - Viscosité apparente relative $\eta_{\mathrm{ra}}$ d'écoulement dans des capillaires de diamètre $D_{t}$, pour différents rapports $D_{t} / D_{c}$ (modèles expérimentaux). Hématocrite $c_{o}=0,40$.

(d'après Sutera et al., 1970).

Lorsqu'au contraire $U \rightarrow 0$, la particule moins déformée tend à occuper la totalité de la lumière du tube, la différence $U_{c}-U$ tendant vers 0 . Sur la figure 2 , sont portées les variations de $U_{c} / U$, pour trois valeurs du rapport $D_{c} / D_{t}$, en fonction d'une vitesse adimensionnelle $V=\eta_{p} U / E D_{t}$ (dépendant de la déformabilité de la particule), définie comme quotient de la contrainte de cisaillement $\eta_{p} U / D_{t}$ par le module d'élasticité $E$ de la membrane ( $\eta_{p}=$ viscosité du plasma).

Associée à cette déformation de la particule, croissante avec $U$, une diminution de la perturbation de l'écoulement a lieu, conduisant à une diminution de la chute de pression $\Delta p$ due à la présence d'une particule. On obtient la diminution de la viscosité apparente $\eta_{a}$. par simple addition de ces effets, dans la limite des faibles hématocrites, limite qui permet de négliger les interactions entre particules. La figure 3 donne $\eta_{a r}$ en fonction de $V=\eta_{p} U / E D_{t}$ pour les mêmes rapports $D_{c} / D_{t}$ que ceux de la figure 2 . Lorsque l'espacement $2 L$ entre les particules n'est plus très grand, on ne peut plus négliger les interactions entre les particules. Mais ces interactions entraînant une immobilisation partielle du fluide (ou tout au moins un ralentissement de l'écoulement) entre deux particules consécutives, on observe une diminution de $\eta_{a r}$. Fung (1969) a déterminé cette réduction, sous la forme d'un facteur "bénéficiaire" $\beta$, fonction du rapport $L / D_{T}$.

Ces résultats confirment les mesures de $\eta_{a r}$ en fonction de $U$ pour des suspensions salines de Globules Rouges, traversant des filtres de polycarbonate (Gregersen et al., 1967). La figure 4 montre ces variations $\eta_{a r}=f(U)$, comparées à celles, simultanées, de $d_{c}$ et de l'épaisseur de la zone annulaire $x=D_{t}-d_{c}$ (déduites des observations de Hochmuth et al., 1970). 


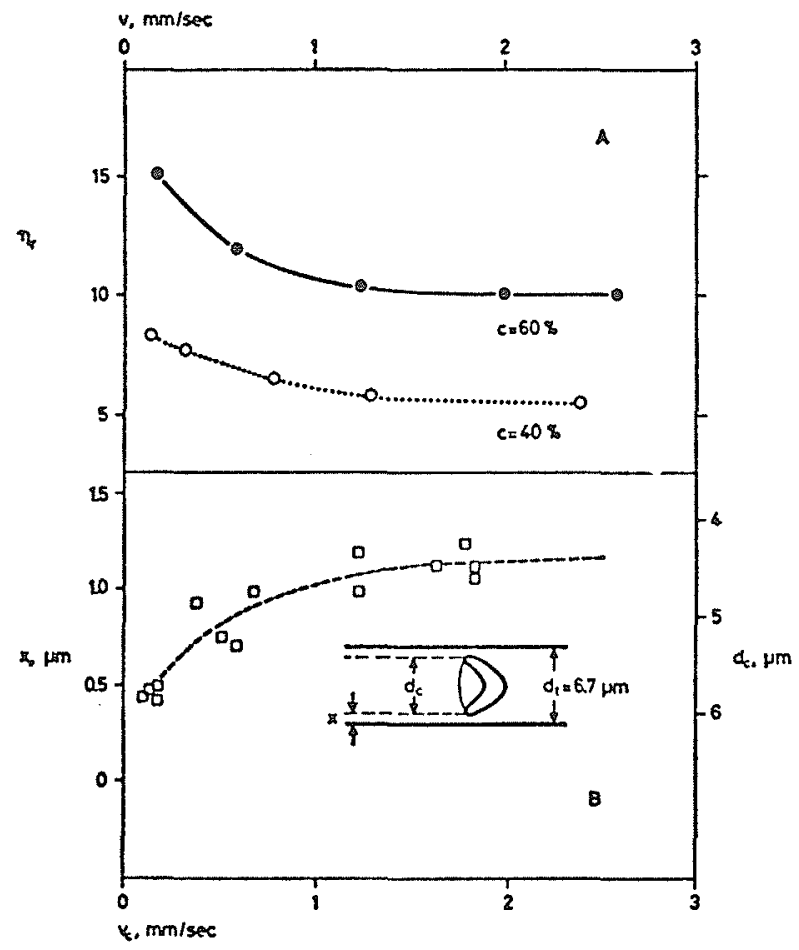

Figure 4.A - Variations de $\eta_{r}$ en fonction de la vitesse moyenne $U$, à trayers un filtre de diamètre moyen de pore $D_{t}=6,8 \mu \mathrm{m}$ (d'après Gregersen et al., 1967).

Figure 4.B - Variations de $x=D_{t}-d_{c}$, en fonction de la vitesse $U_{c}$ des Globules Rouges dans un capillaire de diamètre $6,7 \mu \mathrm{m}$. (d'après Hochmuth et al., 1970). (d'après Chien, 1972)

Lorsque son épaisseur $x$ devient nettement inférieure à $D_{t}$, cette zone annulaire joue le rôle d'une couche lubrifiante. Lighthill (1968), puis Fitzgerald (1969 a et 1969 b) ont développé des modèles basés sur la théorie de la lubrification. Si $p(z)$ est la valeur locale (fonction de la coordonnée $z$, dans le sens de l'écoulement) de la pression dans cette couche, d'épaisseur $h(z)$, cette théorie fournit une première relation entre $p$ et $h$. Une seconde relation est obtenue à partir des lois de comportement du Globule Rouge et (éventuellement) de celle de la paroi du vaisseau. Enfin, une troisième relation relie l'intégrale des forces de pression à la force mécanique (la différence de pression extérieure, appliquée aux deux extrémités du tube) qui met le Globule Rouge en mouvement. Ces trois relations permettent de calculer une épaisseur moyenne $h \approx 0,07 R$ et conduisent à une résistance à l'écoulement qui croît comme $\sqrt{U_{c}}$, conformément à l'expérience (Hochmuth et al., 1970).

\section{b) Augmentation du transport}

\section{b1) Effet du "bolus flow"}

L'expérience de Prothero et Burton (1961) a montré l'augmentation du transport de chaleur à travers les parois d'un tube lorsque celui-ci est traversé par des bulles de gaz (dans l'eau ou l'alcool). Mais ces résultats ne sont pas directement transposables aux conditions in vivo : ils correspondent à des valeurs du nombre de Reynolds $R e \geqslant 10$, bien supérieures à celles existant dans les capillaires $\left(R e \simeq 10^{-2}\right.$ ). Néanmoins, un effet positif devrait être attendu puisque le nombre de Peclet, $P e=U R / D$ (rapport du temps de diffusion(1) $t_{D}=R^{2} / D \sim 10^{-2} \mathrm{sec}$, au temps de recirculation $\left.t_{R}=R / U \sim 10^{-4} \mathrm{sec}\right)$ n'est pas très petit: on a en effet $P e=(\nu / D) R e$, soit, avec $\nu / D \sim 10^{3}, P e \sim 1-10$. Les modèles numériques (Aroesty et Gross, 1970) ne confirment pas cette augmentation due au bolus flow : cela résulte qu'à très faible valeur de $R e$, la recirculation dans l'embol se fait à une vitesse $U^{\prime}$ très inférieure à $U$, donc avec un temps caractéristique $t_{R}^{\prime} \sim R / U^{\prime} \gg t_{R}$, d'où $P e \ll 1$.

\section{b2) Effet de la couche lubrifiante}

Du point de vue du transport, cette couche ne joue aucun rôle effectif ; le temps de diffusion pour la traverser est en effet $t_{-D}^{\prime} \sim h^{2} / D=(h / R)^{2} t_{D}$ soit, avec $h=0,07 R, t_{D}^{\prime} \sim 5.10^{-3} t_{D}$, et ce sont les perméabilités de la membrane du Globule Rouge et de la paroi capillaire qui contrôleront les mécanismes du transport.

\section{La circulation dans les petits vaisseaux}

\section{Caractéristiques générales}

Les vaisseaux concernés(2) ont un rayon $R$ compris entre $5 \mu \mathrm{m}$ et $250 \mu \mathrm{m}$ environ. Le "rayon" des Globules Rouges n'est donc plus négligeable devant le rayon $R$ et les modèles continus devront être utilisés avec prudence notamment pour les plus petites artérioles et vénules $(1 \leqslant(R / a) \leqslant 2)$, où la circulation s'apparente aux écoulements capillaires.

Cette micro-circulation a pour rôle principal d'assurer le transport du sang dans les meilleures conditions; le réseau de vaisseaux constitue un véritable adaptateur d'impédance hydraulique qui garantit :

- la maintenance du débit (vitesse $\sim 1 \mathrm{~mm} / \mathrm{sec}$ ), compatible avec l'apport d'oxygène,

- l'absorption de toutes les surpressions (en particulier, grâce aux anastomoses arteriolo-vénulaires à l'entrée du réseau de capillaires, assez rigides),

- l'absorption de toute la pulsatilité qui serait sans doute peu favorable au mouvement des Globules Rouges dans les capillaires et aux phénomènes de transport associés. Cette absorption se traduit par une véritable diffusion de la pression, analogue à celle qui a lieu dans un milieu poreux, mais qui met en jeu, non seulement la viscosité du fluide, mais aussi la distensibilité des vaisseaux (Lighthill, 1969).

La résistance à l'écoulement dans un tube de rayon $R$ est en général exprimée à l'aide d'une viscosité apparente $\eta_{a}$, définie à partir de la loi de Poiseuille, reliant le débit $Q$ au gradient de pression $\Delta p / L$ :

$$
\mathcal{R}=\frac{\Delta p}{Q}=\frac{8 L}{\pi R^{4}} \eta_{a}
$$

(1) On a pris ici l'exemple de l'Oxygène, avec $D \sim 10^{-5}$ $\mathrm{cm}^{2} \mathrm{sec}^{-1}$.

(2) Ces petits vaisseaux ( $2 a \leqslant R \leqslant 50 a)$ sont les artères terminales $(10 \leqslant R / a \leqslant 50)$, les artérioles $(1 \leqslant R / a \leqslant 10)$, les vénules $(1 \leqslant R / a \leqslant 15)$, les petites veines $(15 \leqslant R / a \leqslant 50)$. 
qui dépend donc de $\eta_{a}$ et de la "géométrie" du tuyau. Dans les expériences in vitro, cette géométrie est gardée constante et $\mathscr{R}$ ne dépend que de $\eta_{a}$. In vivo au contraire, le nombre et les dimensions de ces vaisseaux peuvent changer suivant les différentes conditions physiologiques et/ou pathologiques : néanmoins, le rôle de ces caractéristiques géométriques a été généralement surévalué devant celui de la viscosité $\eta_{a}$. C'est en effet dans les petits vaisseaux que les variations de $\eta_{a}$ avec la concentration et avec le cisaillement se font le plus sentir et entraînent des propriétés sanguines habituellement qualifiées d" "anormales".

Ces propriétés anormales se manifestent au niveau des écoulements, par les caractères généraux suivants :

(i) l'existence d'un régime diphasique avec, au voisinage de la paroi du tube, une zone marginale ("couche plasmatique") appauvrie en particules, et par suite, une région axiale enrichie ("noyau central") ;

(ii) l'aplatissement du profil de vitesse au voisinage de l'axe;

(iii) la diminution de la concentration moyenne $c_{t}$ circulant dans le tube, comparée à la concentration moyenne $c_{o}$ du "réservoir" d'alimentation (Effet Farhaeus);

(iv) la diminution de la viscosité apparente $\eta_{a}$ au fur et à mesure que le rayon $R$ du tube diminue (Effet Farhaeus-Lindqvist).

Ces propriétés, observées dans les écoulements sanguins, sont aussi rencontrées dans un grand nombre d'écoulements de dispersions concentrées.

Il est habituel, pour l'hydrodynamicien, d'étudier les propriétés du fluide - c'est-à-dire ici, l'hémorhéologie avant de vouloir en décrire les propriétés en écoulement. Nous décrirons donc d'abord les propriétés de la viscosité globale du sang.

\section{Viscosité du sang}

a) En régime stationnaire, la viscosité apparente $\eta_{a}$ du sang (additionné d'un anticoagulant) telle qu'on la mesure dans un viscosimètre (Couette ou capillaire), est trouvée fonction de la concentration moyenne $c_{o}$ et de la vitesse apparente de cisaillement $\dot{\gamma}_{a}\left(\eta_{p}=\right.$ viscosité du plasma) :

$$
\begin{gathered}
\eta_{a r}=\frac{\eta_{a}}{\eta_{p}}=\eta_{a r}\left(c_{o}, \dot{\gamma}_{a}\right), \\
\operatorname{avec} \frac{\partial \eta_{a}}{\partial c_{o}}>0 \text { et } \frac{\partial \eta_{a}}{\partial \gamma_{a}}<0
\end{gathered}
$$

La figure 5 empruntée à S. Chien et al. (1967) montre ces variations, pour du sang normal et pour une suspension de Globules Rouges dans une solution saline (1iquide de Ringer) et prouve que l'essentiel de la variation en température $T$ est contenu dans celle de la viscosité du plasma.

Dans la mesure où l'on suppose l'existence d'une viscosité locale définie en chaque point du fluide (dépen-

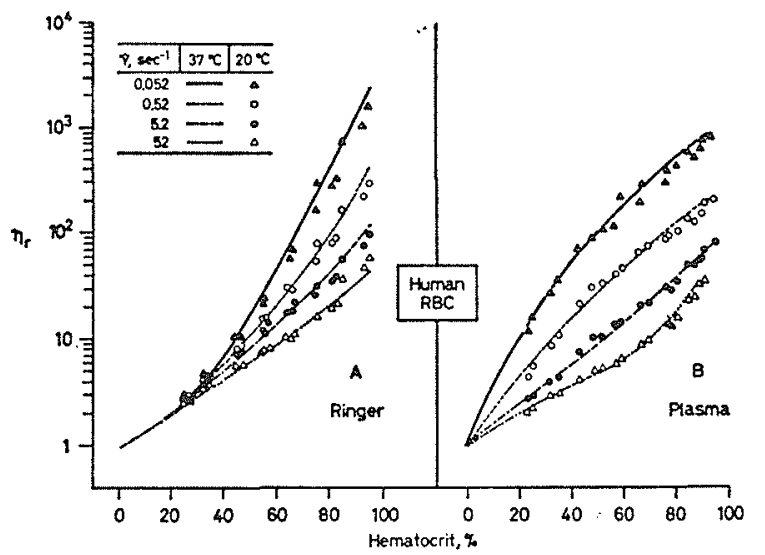

Figure 5 - Viscosité apparente relative en fonction de la concentration volumique et de la vitesse de cisaillement $\dot{\gamma}$ (globules rouges humains, en suspension dans du liquide de Ringer (A) et dans du Plasma (B)). Mesures à $20^{\circ} \mathrm{C}$ (Points) et à $37^{\circ} \mathrm{C}$ (Courbes) :

(d'après Chien et al., 1971).

$$
\eta_{p}\left(20^{\circ} \mathrm{C}\right) / \eta_{p}\left(37^{\circ} \mathrm{C}\right)=1,5
$$

dant des propriétés locales de la suspension), il est généralement admis que cette viscosité locale suit une loi analogue à (2) :

$$
\eta_{r}=\frac{\eta}{\eta_{p}}=\eta_{r}(c, \dot{\gamma})
$$

$c$ et $\dot{\gamma}$ étant les valeurs locales de la concentration et du cisaillement de vitesse. Cependant, la plupart des milieux dispersés montrent une viscosité qui dépend de leur structure, laquelle dépend de $c$ et $\dot{\gamma}$ (viscosité structurelle d'Ostwald). Dans le cas du sang, cette structure pourra être globalement caractérisée par l'état d'agrégation (aux faibles $\dot{\gamma}$ ) et de déformation (aux forts $\dot{\gamma}$ ) des Globules Rouges. Dans des conditions données, les paramètres figurant dans la relation (3) dépendront donc de l'état structurel de la suspension. En retour, on peut donc s'attenđre à ce que (2) contienne, elle aussi, des paramètres dépendant de cette structure, et par suite, puisse être utilisée pour la caractérisation du système.

Un très grand nombre de lois (3) ont été proposées. En régime newtonien (c'est-à-dire à fort débit), les lois $\eta_{r}=f(c)$ sont connues en milieu très dilué, par extension de la loi d'Einstein $\eta_{r}=1+2,5 c$, établie pour les suspensions de spheres dures. Elles ont été appliquées au sang avec plus ou moins de succès. La relation d'Oliver et Ward (1953) :

$$
\eta_{r}=\frac{1}{1-k c}
$$

semble ètre bien vérifiée (Maude et Whitmore, 1956) pour des concentrations moyennes $(c \leqslant 0,30)$, mais avec un coefficient $k$ (viscosité intrinsèque) empirique, qui intervient comme paramètre de structure (dépendant en particulier de la forme des particules).

A plus faible débit, le sang révèle un comportement non-newtonien. La loi de Casson (1959):

$$
\begin{aligned}
& \eta^{1 / 2}=\frac{\tau^{1 / 2}}{\dot{\gamma}^{1 / 2}}=K^{1 / 2}+\frac{\tau_{0}^{1 / 2}}{\dot{\gamma}^{1 / 2}} \\
& (\tau=\eta \gamma=\text { contrainte de cisaillement })
\end{aligned}
$$




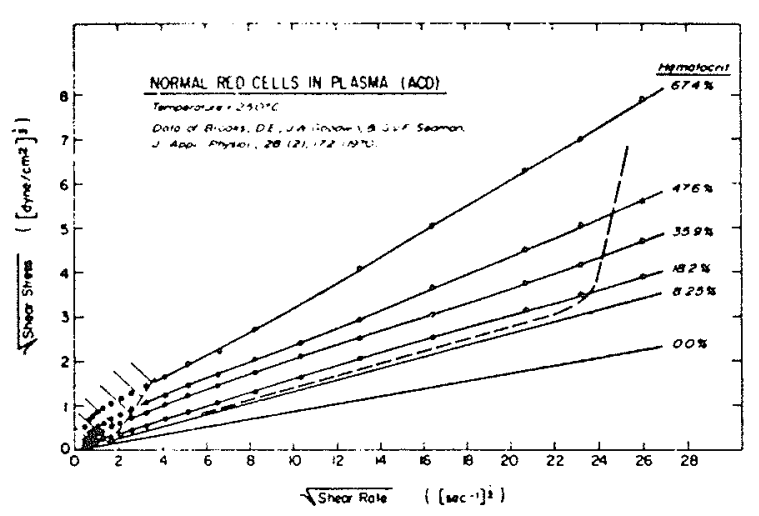

Figure 6 - Diagramme de Casson pour du sang (additionné d'anticoagulant ACD). (Pour la clarté, les points pour $H=0$ et $H=8,25$ n'ont pas été reportés : ils correspondent à des fluides new toniens).

(d'après Cokelet, 1972).

établie pour des suspensions de pigments s'agrégeant en bâtonnets (encres d'imprimerie) est bien vérifiée par le sang, dans un très large domaine de concentration et de $\dot{\gamma}$, excepté au voisinage de $\dot{\gamma} \rightarrow 0$, où il ne semble pas que l'on observe de contrainte seuil $\tau_{o}$. La figure 6 montre cet accord et précise les variations avec $c$ de la viscosité de Casson $K$ et de la contrainte seuil $\tau_{0}$. L'influence des protéines plasmatiques, notamment le fibrinogène, a été associée, à travers l'agrégation des Globules Rouges, à ces effets non newtoniens (Merrill et al., 1965).

D'autres lois phénoménologiques $\eta=f(\dot{\gamma})$, analogues à celles qui ont été développées pour les fluides nonnewtoniens (colloïdes, polymères...) pourraient être utilisées. Mais de telles lois dépendent de paramètres phénoménologiques dont le sens physique est souvent difficile à préciser, et, à travers les relations comportement-structure, dépendent fondamentalement du type d'écoulement étudié. Aussi, il semble préférable d'effectuer d'abord l'analyse détaillée des propriétés des écoulements, et de tenter de préciser, ensuite, un modèle de comportement global, caractérisé, en régime stationnaire, par la viscosité non-newtonienne (3).

b) D'autres propriétés (électroviscosité, par ex.) et des effets dépendants du temps (thixotropie, viscoélasticité) présentés par un grand nombre de milieux dispersés, ont été observés dans le cas du sang, mais leur étude sortirait du cadre de cet exposé.

Propriétés des écoulements dans les petits vaisseaux

\section{Ecoulement diphasique annulaire}

L'existence de la couche plasmatique a été très discutée mais est maintenant bien établie. Des études directes du mouvement de particules individuelles (Goldsmith et Mason, 1967) ont prouvé le processus de migration radiale des particules, dans des conditions bien définies (dépendant de la rigidité des particules, mais très peu de leur forme) et d'un nombre de Reynolds interne $\left.R_{i}=\rho a^{2} \dot{\gamma} / \eta_{p}\right)$.
A concentration élevée, il est fort probable que ces (faibles) forces de migration soient largement contrebalancées par celles résultant de l'entassement des particules. Quoiqu'il en soit, un effet purement stérique entraîne une diminution de concentration au voisinage de la paroi (Effet Vand, 1948), sur une épaisseur de l'ordre de $a$, rayon de la particule : cette diminution imposera une discontinuité dans le profil de concentration $c(r)$ de tout modèle continu (Whitmore, 1968). Néanmoins, les mesures de ces profils, à forte concentration, sont très difficiles et une première approximation sera de supposer constantes les concentrations dans les deux phases.

Les travaux de Buggliarello et al. (1965) ont montré le caractère aléatoire de la position de la limite de la couche plasmatique; son épaisseur moyenne $\delta$ a été trouvée fonction, à la fois, de l'hématocrite, de la contrainte $\tau_{R}$ appliquée, et du rayon du tube $R$ (Fig. 7). Elle doit dépendre aussi de la forme et de la déformabilité des particules.
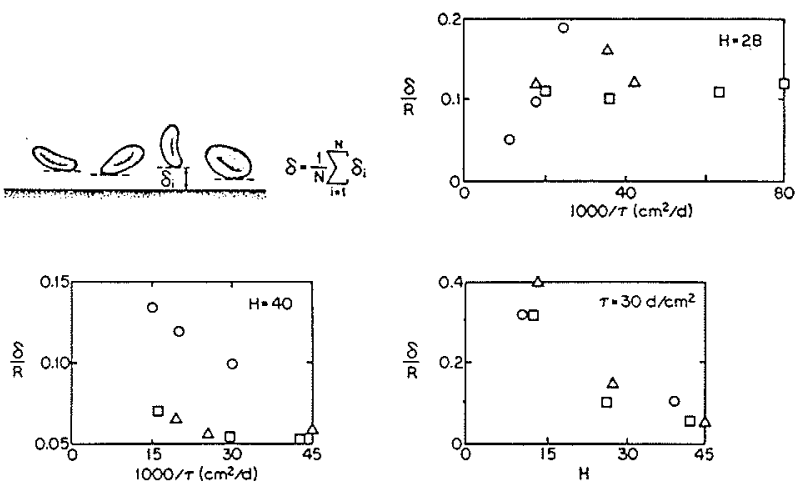

Figure 7 - a) Définition statistique de l'épaisseur $\delta$ de la couche plasmatique. b), c) et d) Variations de $\delta / R$ en fonction de l'inverse de la contrainte $\tau_{R}$ et de l'hématocrite $H$, pour trois capillaires de rayons $R=20 \mu \mathrm{m}(\mathrm{o}), 33,6 \mu \mathrm{m}(\Delta)$ et $41,5 \mu \mathrm{m}$ (a). (d'après les observations directes de Buggliarello et al., 1965).

\section{Profil de vitesse aplati}

(Profil partiellement "bouchon")

La présence de la couche plasmatique, associée à un profil de concentration $c(r)$, a pour première conséquence l'existence d'un écoulement diphasique annulaire, avec une phase centrale (s) et une phase périphérique $(w)$ occupant respectivement les domaines $o \leqslant r \leqslant \beta R$ et $\beta R \leqslant r \leqslant R$. Mais on doit tenir compte des propriétés générales de la viscosité. Puisque $(\partial \eta / \partial c)>0$, la phase centrale ( $s$ ) est plus visqueuse que la phase périphérique $(w)$ : le profil des vitesses est donc aplati dans la région centrale. Cet aplatissement est exacerbé par l'augmentation de $\eta$ quand $\dot{\gamma}$ diminue, $(\partial \eta / \partial \dot{\gamma})<0$. A forte concentration, et pour des débits assez faibles, on doit donc s'attendre à des profils assez abrupts, avec un noyau très visqueux, entourée d'une mince couche peu visqueuse (qui a été qualifiée de couche lubrifiante).

La figure 8 montre de tels aplatissements, pour différentes conditions (débits, concentration, rapport $a / R$ ) (Karnis et al., 1966). 

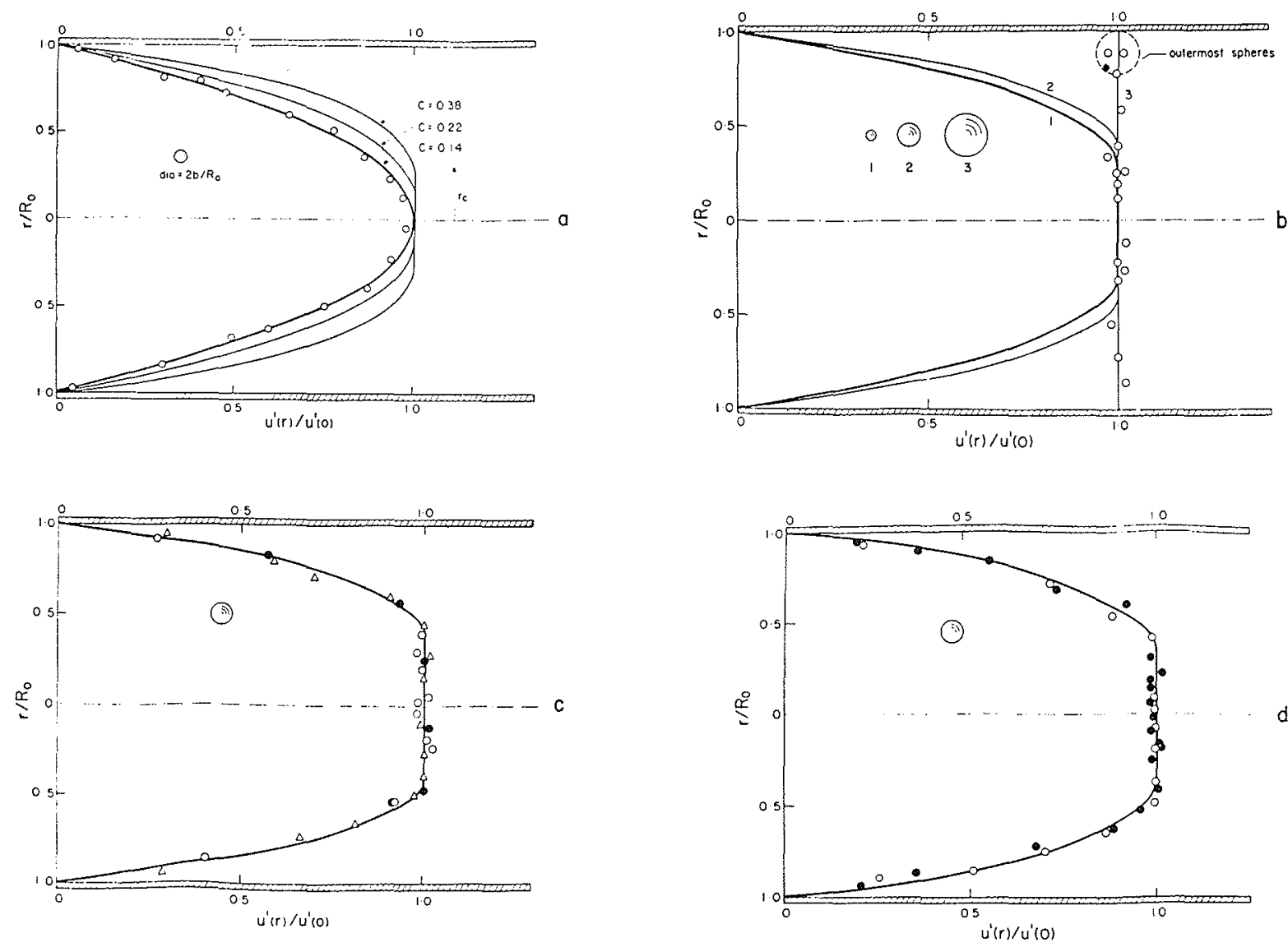

Figure 8 - Profils de vitesse dans des écoulements de suspensions de sphères rigides (diamètres $2 a$ ) dans des tubes de rayon $R_{o}$. (la dimension relative des sphères est montrée)

a) Effet de la concentration (chaque courbe est expérimentale ; pour $c=0,14$ c'est une parabole). On voit l'aplatissement partiel, de ray on $r_{b}$

b) Effet de la taille des particules : (1) $c=0,32, a / R_{o}=0 ; 026$; (2) $c=0,34, a / R_{o}=0,056$; (3) $c=0,34, a / R_{o}=0,112$.

c) Effet du débit $Q$, ̀̀ $c=0,34$ et $a / R_{o}=0,056$. (๑) $Q=7,11 \mathrm{~mm}^{2} \mathrm{sec}^{-1} ;(\mathrm{o}) Q=35,6 \mathrm{~mm}^{3} \mathrm{sec}^{-1} ;(\Delta) Q=71,1 \mathrm{~mm}^{3} \mathrm{sec}^{-1}$

d) Comparaison des vitesses des particules (sphères de polystyrène, (o)) et du fluide (particules d'Aluminium, comme traceurs : ( ()$\left.^{)}\right)$.

(D'après Karnis et al., 1966).

\section{Effet Fahraeus (Fahraeus, 1929)}

Une seconde conséquence de l'écoulement diphasique est l'inégalité de la concentration moyenne instantanée $c_{t}$ dans le tube, et de la concentration débitante $c_{d}$. Si l'on suppose les concentrations des deux phases $c_{s}=C^{t e}$ et $c_{w}=0$, la concentration moyenne est :

$$
c_{t}=\left(\pi R^{2}\right)^{-1} \int_{0}^{R} c 2 \pi r d r=\beta^{2} c_{s}
$$

et le bilan du transport de particules ${ }^{(3)}$ s'écrit :

$$
c_{d}=\int_{0}^{R} c \nu 2 \pi r d r / \int_{0}^{R} \nu 2 \pi r d r=\beta^{2} c_{s} \frac{U_{s}}{U}
$$

$U$ et $U_{s}$ désignant respectivement la vitesse moyenne totale et celle de la phase $(s):$ d'où $c_{t}<c_{d}$ puisque $U$ est toujours inférieure à $U_{s}$.

L'inégalité de la concentration débitante $c_{d}$ ("hématocrite de décharge") et de celle $c_{o}$ du réservoir d'ali-

(3) Dans chaque phase, on néglige toute différence de vitesse entre le fluide et les particules, conformément à l'observation (Karnis et al, 1966). mentation a été supposée associée à un réarrangement de la distribution de concentration à l'entrée du tube, effet qui dépend donc du débit (Cokelet, 1976). Néanmoins, cet effet n'est sensible qu'aux très faibles valeurs de $R$ telles que $(R / a) \sim 2-3$. Pour les valeurs supérieures, $R \geqslant 3 a$, on pourra, en première approximation, négliger cette différence. Dans toute la suite, on posera donc $c_{d}=c_{o}$, d'où :

$$
c_{t}=f\left(c_{o}, R\right)
$$

L'inégalité entre $c_{t}$ et $c_{o}$ a été observée pour la première fois par Fahraeus en 1929. La figure 9 montre la variation du rapport $c_{t} / c_{o}$ en fonction de $c_{o}$ pour différentes valeurs du rayon $R$ du tube (Barbee et Cokelet, 1971 a).

\section{Effet Fahraeus-Lindqvist}

(Fahraeus-Lindqvist, 1931)

De très nombreus travaux ont été consacrés à l'étude de cet effet. La figure 10 reproduit les résultats des mesures de Haynes et Burton (1959), pour $0,2 \leqslant c_{o} \leqslant 0,8$ 


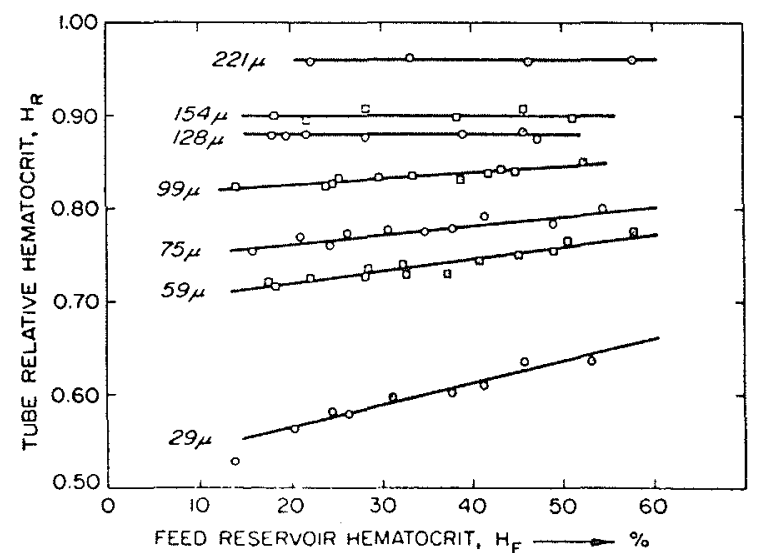

Figure 9 - Effet Fahraeus : variations du rapport $c_{t} / c_{o}$ en fonction de $c_{o}$ et du diamètre $D_{t}$ du tube

(d'après Barbee et Cokelet, 1971).

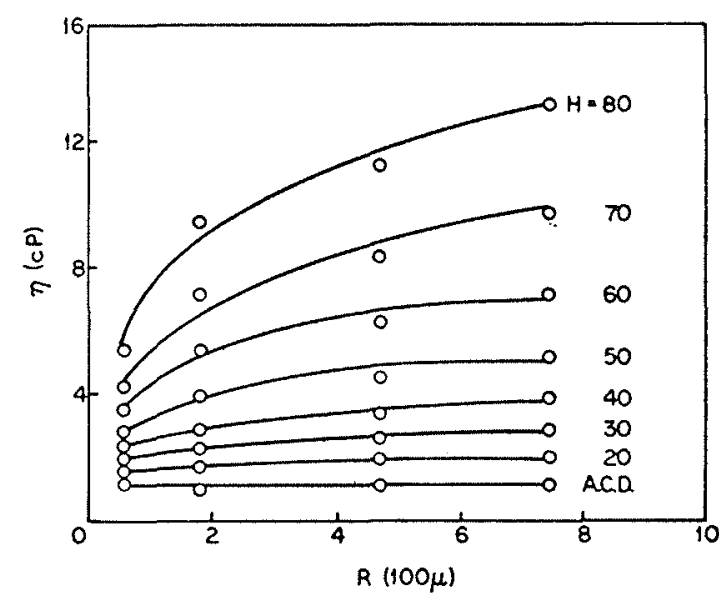

Figure 10 - Effet Fahraeus-Lindqvist, $\eta_{a}=f(R)$, pour différents hématocrites $H$.

(d'après Haynes, 1961)

et dans des tubes longs ( $L / R \sim 90$ : effets d'entrée négligeables) de rayon $R$ tel que $67 \mu \mathrm{m} \leqslant R \leqslant 750 \mu \mathrm{m}$. Cette diminution de $\eta_{a}$, quand le rayon $R$ décroît, est d'autant plus importante que la concentration est élevée.

Le même type de résultats, exprimés sous la forme ${ }^{(4)}$ $\tau_{R}=f(\dot{\gamma} a)$, obtenus par Barbee et Cokelet (1971 b), pour un tube de $29 \mu \mathrm{m}$, à différents hématocrites, sont présentés sur la figure 11 . Ils montrent aussi la diminution de $\eta_{a}$ lorsque $R$ décroît, à $H=C^{t e}$. Ces auteurs ont comparé :

(i) les valeurs mesurées $\eta_{a}\left(c_{o}, R\right)$ (dans ce tube de rayon $R=29 \mu \mathrm{m}$ et pour une concentration $c_{o}$ du réservoir), et

(ii) les valeurs calculées à l'aide de la relation générale $\eta_{a}^{\infty}(c)$ établie expérimentalement dans les tubes de "grand" diamètre $(R \geqslant 250 \mu \mathrm{m})$ lorsqu'on y remplace la concentration du réservoir par celle du tube.

(4) qui n'est autre que la loi de Poiseuille, avec la contrainte pariétale $\tau_{\mathrm{R}}=\frac{R}{2} \frac{\Delta p}{L}$ et le cisaillement apparent $\dot{\gamma}_{a}=\frac{4 Q}{\pi R^{3}}$.
Ils ont constaté que l'égalité :

$$
\eta_{a}\left(c_{o}, R\right)=\eta_{a}^{\infty}\left(c_{t}\right), \text { avec } c_{t}=f\left(c_{o}, R\right)
$$

est remarquablement vérifiée (Fig. 11). Ce résultat, assez surprenant puisqu'il implique l'explication de l'effet Fahraeus-Lindqvist par le seul effet Fahraeus, n'a pas encore reçu de confirmation théorique.

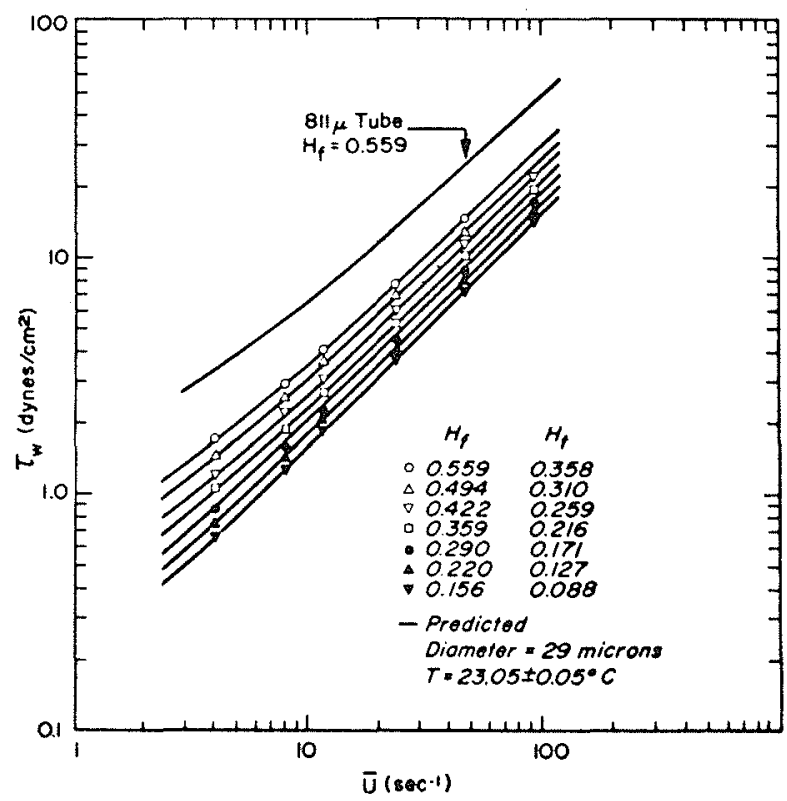

Figure 11 - Variations $\tau_{R}=f(\vec{U})$ dans un tube de diamètre $D_{t}=29 \mu \mathrm{m}$. Chaque groupe de points mesurés se placent sur la courbe $\tau_{R}=f\left(\vec{U}, c_{o}\right)$ obtenue pour les tubes de grands diamètres, quand on y remplace $c_{o}$ par $c_{t}$. (d'après Barbee et Cokelet, 1971).

Rappelons, que lorsque $R$ décroît, $\eta_{a}$ ne décroît pas sans limite : vers $R \sim 2 a, \eta_{a}$ passe par un minimum, puis $R$ continuant de diminuer, $\eta_{a}$ croît en se raccordant à la variation rencontrée dans la circulation capillaire (Cf. Fig. 3),

De tels effets ont été observés dans d'autres suspensions (Cf. par ex., Seshadri et Sutera, 1970).

\section{Modèles diphasiques}

Le but de tels modèles est de décrire de la manière la plus cohérente possible les propriétés précédentes. En première approximation, on y suppose la phase périphérique constituée de fluide pur, donc $c_{w}=0$.

Un très grand nombre de travaux, s'appuyant sur différents modèles (effet sigma, glissement à la paroi) ont tenté de justifier théoriquement l'existence de l'effet Fahraeus-Lindqvist, et d'en déduire la valeur de l'épaisseur de la couche plasmatique $\delta=(1-\beta) R$ et l'aplatissement du profil de vitesse. Nous nous limiterons ici aux modèles diphasiques les plus classiques que nous regrouperons pour en dégager les caractères essentiels. 


\section{A-Couche plasmatique}

1) Le modèle "bi-newtonien" est le plus simple. En supposant les deux phases newtoniennes, avec des viscosités constantes $\eta_{s}$ et $\eta_{P}$, le calcul du débit à l'aide de l'équation de Navier-Stokes (avec la condition de nonglissement à la paroi) donne la viscosité apparente :

$\eta_{a}=\eta_{P}\left[1-\left(1-\frac{\delta}{R}\right)^{4}\left(1-\frac{\eta_{P}}{\eta_{s}}\right)\right]^{-1}$ où $\frac{\delta}{R}=1-\beta$

1.a) en supposant l'épaisseur $\delta$ de la couche plasmatique très petite devant $R$ (hypothèse valable aux fortes concentrations), on a, au premier ordre en $\delta / R$, après réarrangement de $(10)$ :

$$
\eta_{a}=\eta_{s}\left[1-4 \frac{\delta}{R}\left(\frac{\eta_{s}}{\eta_{p}}-1\right)\right]
$$

Si $\delta$ ne dépend que de l'interaction particule-paroi, elle doit être indépendante de $R$ lorsque $(\delta / R) \ll 1$. La relation (11) explicite alors l'effet FahraeusLindqvist $\eta_{a}=f(R)$. La confrontation de (11) aux mesures de Fahraeus-Lindqvist (1931) (pour $c=0,411,20 \mu \mathrm{m} \leqslant R \leqslant 250 \mu \mathrm{m}$ et $80 \leqslant \tau_{R} \leqslant$ 160 dynes $\mathrm{cm}^{-2}$ ) est présentée sur la figure 12 . A partir de la pente et de l'ordonnée à l'origine, pour les faibles valeurs de $1 / \mathrm{R}$, il est possible de calculer $\delta$. On obtient $\delta \simeq 1-1,3 \mu \mathrm{m}$.

1.b) Haynes (1960) a ajusté la relation (10) sur les mesures données sur la figure 10 . La figure 13 montre les variations $\delta=f(H)$. Pour les valeurs normales de $H$, on obtient $\delta \simeq 3 \mu \mathrm{m}$.

2) Cependant, l'effet Fahraeus a été négligé dans ce qui précède. Sous l'hypothèse de l'unicité de la fonction $\dot{\gamma}=f(\tau)$, on dispose de deux relations thériques explicitant respectivement l'effet Fahraeus-Lindqvist et l'effet Fahraeus. La première (identique à $(10)$ ) relie la fluidité

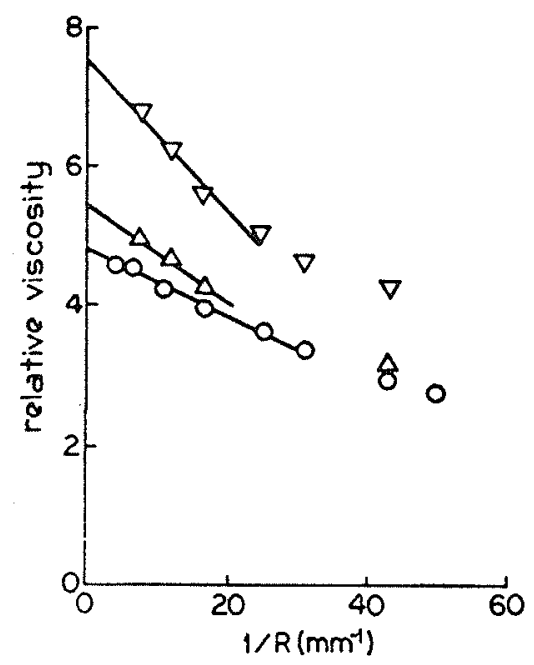

Figure 12 - Ajustement de la relation (11) aux mesures de Fahraeus-Lindqvist (1931). (d'après Middleman, 1972).

(5) exprimée ici en fonction de $\tau$ au lieu de $\dot{\gamma}$, ce qui est équivalent si $\dot{\gamma}=f(\tau)$ est uniforme.

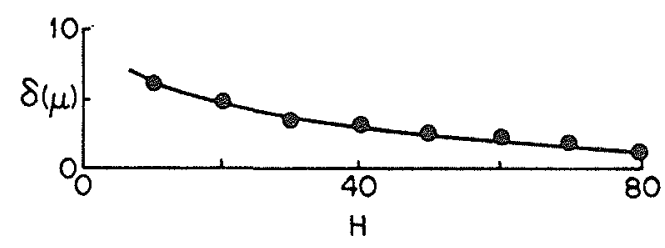

Figure 13 - Ajustement de la relation (10) aux mesures de Haynes, 1961 (Cf. Fig. 10).

(d'après Haynes, 1961).

relative apparente $\varphi_{a}=\eta_{P} / \eta_{a}=\varphi_{a}\left(c_{o}, \tau_{R}, R\right)$ à la fluidité relative locale (5). La seconde exprime la conservation du débit de particules (Cf. (6)). Dans le cas de l'écoulement diphasique caractérisé au paragraphe précédent, ces relations s'écrivent (Thomas, 1961)

$$
\begin{gathered}
\varphi_{a}=1-\beta^{4}\left(1-\varphi_{s}\right) \\
\frac{c_{t}}{c_{o}} \# \frac{c_{t}}{c_{d}}=\frac{\beta^{2} c_{s}}{c_{d}}=\frac{1-\beta^{4}\left(1-\varphi_{s}\right)}{2\left(1-\beta^{2}\right)+\beta^{2} \varphi_{s}}
\end{gathered}
$$

où $\varphi_{s}=\varphi\left(c_{s}, \beta \tau_{R}\right)$

2.a) Thomas (1961) a utilisé une méthode d'approximations successives pour résoudre simultanément (12) et (13). A partir des données de Kumin (1949) et de Bayliss (1952), il obtient $\delta=f\left(\tau_{R}, R\right)$ à $c_{o}=0,40$, avec des valeurs typiques $\delta \sim 1,5 \mu \mathrm{m}$ à $\tau_{R} \rightarrow 0$ et $3,8 \leqslant \delta \leqslant 5 \mu \mathrm{m}$ à $\tau_{R} \rightarrow \infty$, montrant des variations de $\delta$ et de $R$ dans le même sens. Mais l'utilisation des données de Fahraeus (1929) (à grand $\tau_{R}$ ), qui donne $\zeta \leqslant \delta \leqslant 9 \mu \mathrm{m}$, semble indiquer une variation inverse.

2.b) Cependant, utilisant la relation newtonienne $\varphi_{s}=1-k c_{s}$ (Cf. (4)) avec $k=2,35$, la même analyse des données de Maude et Whithmore (1956) [suspensions de sphères dures, $a=48 \mu \mathrm{m}$, dans des tubes larges, $R=938 \mu \mathrm{m}]$ donne, pour $0,16 \leqslant c_{o} \leqslant 0,20$ et $\tau_{R}$ grand, $\delta=0,78 a$ c'est-à-dire $\delta$ \# Cte, (Thomas, 1961).

Les résultats de Thomas ne sont pas en bon accord avec les observations de Bugliarello et al. (1965), portées sur la figure 7 . De plus, l'utilisation d'une relation newtonienne telle que (4) n'est pas satisfaisante puisque même à fort $\tau_{R}$, la région près de l'axe, où $v(r)$ est aplati,

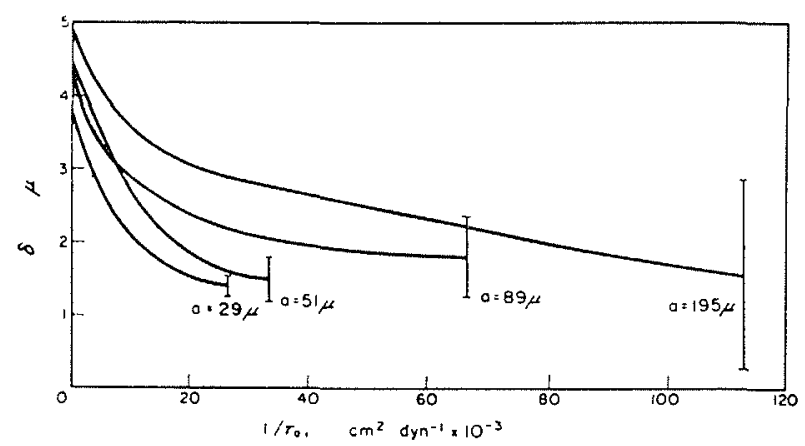

Figure 14 - Résultats de Thomas (1961) obtenus par ajustement de (12) et (13) aux données de Kumin (1949), montrant les variations de $\delta$ avec $R$ et $\tau_{R}$ (Hématocrite $=40$ ).

(d'après Thomas, 1961). 
contient des faibles valeurs de $\gamma_{s}$ et exige l'utilisation d'une relation non-newtonienne $\eta=f(c, \dot{\gamma})$.

3) Charm et al. (1968) ont utilisé une telle relation non-newtonienne, la relation (5) de Casson. Après avoir déterminé, par viscosimétrie Couette, les constantes $k$ et $\tau_{o}$ qui y figurent, ils utilisent les mesures de $\varphi_{a}$ en viscosimétrie capillaire (sous forme de la relation pressiondébit) pour déterminer $\delta / R$. La figure 15 illustre ces résultats, qui montrent une assez grande dispersion, sans doute due à la non-validité de (5) à faible $\dot{\gamma}$.

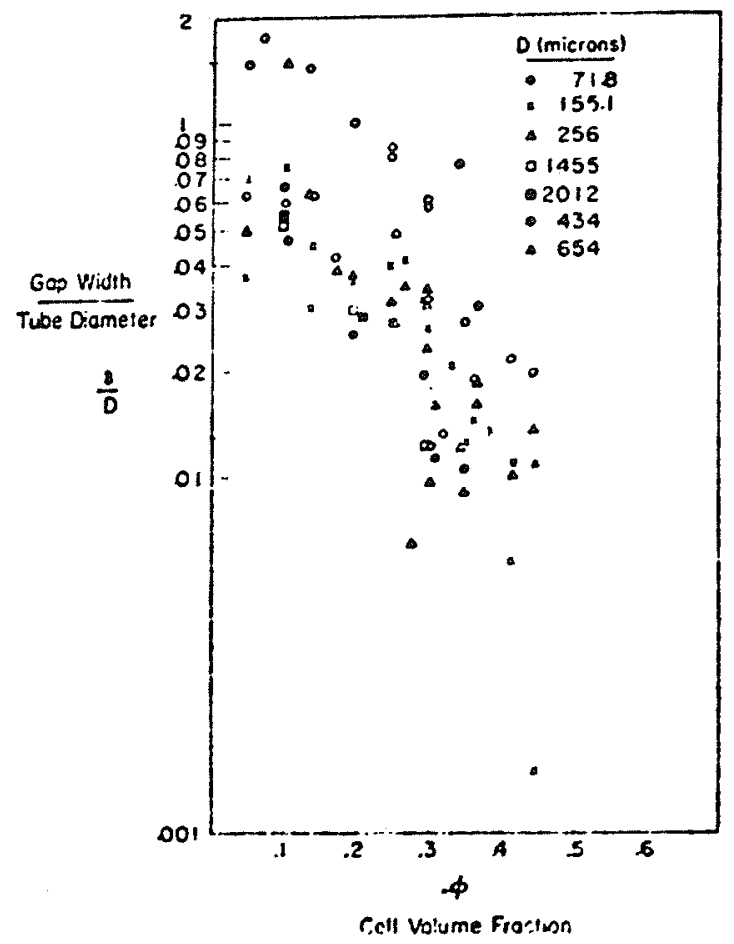

Figure 15 - Variations $\delta / D_{t}$ en fonction de la concentration volumique $c$, obtenues dans le cas du modèle newtonien-Casson. (d'après Charm et al., 1968).

4) Lorsqu'on dispose, en plus des mesures de $\varphi_{a}$, de celles du rapport $c_{t} / c_{o}$, on peut, en supposant $c_{d} \# c_{o}$, déterminer $\beta$ en éliminant $\varphi_{s}$ entre les deux équations (12) et (13). Malheureusement, il n'existe que peu de données "complètes" : lorsqu'on connait $c_{t} / c_{o}$, on ne connait pas en général les valeurs de $\varphi\left(\tau_{R}\right)$ pour la même expérience, et, à ce jour, les différentes tentatives effectuées n'ont pas donné de résultats très concluants quant aux variations $\delta\left(c_{o}, \tau_{R}, R\right)$. Le principal intérêt des modèles diphasiques est de montrer que, dans $\eta_{a}$, l'essentiel de la dépendance en $R$ est contenu dans celle de l'épaisseur de la couche plasmatique, c'est-à-dire dans $\beta$.

\section{$B$ - Profils de vitesse}

Tous les modèles diphasiques précédents conduisent à un aplatissement du profil $v(r)$ puisque la phase centrale est plus visqueuse que la couche périphérique. Qualitativement ces modèles sont donc satisfaisants, mais ils n'ont pas donné, jusqu'à présent, de résultats quantitativement acceptables. De plus le concept de viscosité structurelle empêche d'identifier, a priori, l'extension radiale de la phase centrale et celle de la région à profil $v(r)$ aplati (rendant donc difficiles les déterminations indirectes de $\delta$ à partir des mesures de ce profil).

a) Le modèle bi-newtonien donne un aplatissement d'autant plus fort, mais avec un raccordement d'autant plus anguleux, que le rapport $\eta_{s} / \eta_{p}$ est grand. De tels raccordements n'ont cependant été observés que lorsque $a / R$ est grand.

b) Le modèle newtonien-Casson est plus satisfaisant puisque ce raccordement est beaucoup plus doux. Mais les valeurs de la contrainte seuil $\tau_{o}$, déduites par viscosimétrie, sont beaucoup trop faibles et donnent un rayon de la partie aplatie $r_{b}=R \tau_{o} / \tau_{R}$, beaucoup trop petit quand on le compare aux rayons observés (Cf. Fig. 8).

Un modèle diphasique à dissipation optimale d'énergie

Ce qui précède montre que pour les milieux dispersés, la viscosité apparente ne peut pas être définie comme une seule fonction des propriétés intrinsèques du fluide, définies par des variables d'état, mais qu'elle dépend aussi des conditions de l'écoulement (en particulier de l'existence de la couche périphérique appauvrie en particules). Bien plus, l'écoulement, qui induit des variations de structure du milieu, réagit sur ces propriétés intrinsèques et la viscosité locale elle-aussi ne peut être définie que pour des conditions d'écoulement fixées. Cela correspond à la notion de viscosité structurelle, qui traduit les propriétés non-newtoniennes du milieu (thixotropie, notamment).

\section{$A-V i s c o s i t e$}

1. L'application du principe de minimisation de l'énergie dissipée par viscosité dans un système diphasique annulaire (avec deux concentrations supposées constantes), en écoulement viscosimétrique simple, a permis, à l'aide d'une méthode variationnelle, d'expliciter une relation $\eta_{r}(c)$ (Quemada, 1975, 1977)

$$
\eta_{r}=\left(1-\frac{1}{2} \overrightarrow{k c}\right)^{-2}
$$

où $\bar{k}$ est une viscosité intrinsèque effective. Cette relation s'applique dans le domaine newtonien, et pour des concentrations très élevées (allant jusqu'à la concentration d'entassement maximum). La figure 16 montre un assez bon accord avec différents groupes de données expérimentales.

2. La prise en compte des changements de structure, notamment ceux résultant de la dispersion des agrégats de particules sous l'effet du cisaillement $\dot{\gamma}$ a permis d'étendre cette relation au domaine non-newtonien (Quemada, 1978). Pour le sang, ces effets nonnewtoniens ont été associés d'une part, à l'agrégation (à faible $\dot{\gamma}$ ) et, d'autre part, à la déformation et l'orientation (à fort $\dot{\gamma}$ ) des Globules Rouges (Cf. Fig. 17 d'après Chien, 1970). Elle conserve la même forme que la rela- 


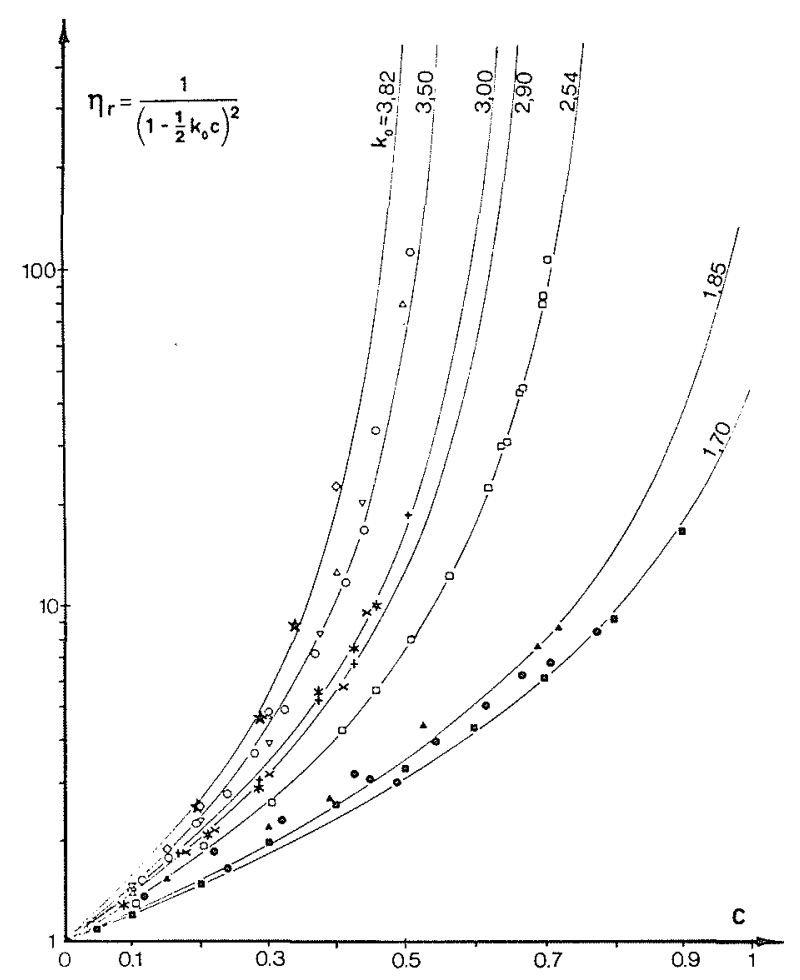

Figure 16 - Viscosité relative $\eta_{r}$ en fonction de la concentration en volume $c$. Courbes : relation (14) $\eta_{r}=\left(1-\frac{1}{2} \bar{k} c\right)^{-2}$, pour différentes valeurs $\vec{k}$. Points expérimentaux : sphères dures $\square$ : $+, *, x ; \nabla, \Delta, \diamond ;$ Particules de latex $o$; Sang et suspensions de globules rouges $₫, \Delta, ;$ globules rouges rigides (anémie falciforme) .

(d'après Quemada, 1977).

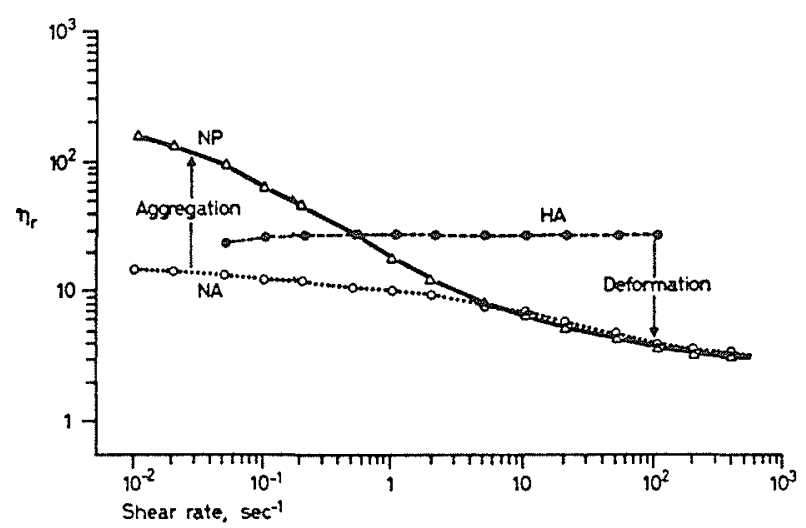

Figure 17 - Viscosité relative $\eta_{r}$ en fonction de la vitesse de cisaillement $\dot{\gamma}$, pour trois suspensions de globules rouges $(H=45$, $\left.\eta_{p}=1,2 c P\right)$.

$N P$ (GR normaux dans du plasma)

$N A$ (GR normaux dans du Ringer + Albumine)

$H A$ ( $G R$ durcis dans du Ringer + Albumine)

(d'après Chien, 1970).

tion (14) précédente, mais avec une viscosité intrinsèque $k$ dépendant de la vitesse de cisaillement réduite $\dot{\gamma}_{r}$

$$
\begin{array}{r}
\eta_{r}=\left(1-\frac{1}{2} k c\right)^{-2}, \operatorname{avec} k\left(\dot{\gamma}_{r}\right)=\frac{k_{o}+k_{\infty} \dot{\gamma}_{r}^{1 / 2}}{1+\dot{\gamma}_{r}^{1 / 2}}, \\
\dot{\gamma}_{r}=\frac{\dot{\gamma}}{\dot{\gamma}_{c}}=\frac{\eta_{r} a^{3} \dot{\gamma}}{K T}
\end{array}
$$

où $k_{o}, k_{\infty}$ et $\dot{\gamma}_{c}$ sont des paramètres définissant globalement l'état structurel du milieu. Leur utilisation pour la caractérisation des milieux dispersés concentrés a été proposée, en particulier pour le sang (Quemada, 1976), où les paramètres $k_{o}$ et $k_{\infty}$ sont respectivement associés à l'agrégation et à la déformation (et l'orientation) des Globules rouges, tandis que $\dot{\gamma}_{c}$ dépend de la taille moyenne des agrégats. Un exemple de variation $\eta_{r}(\dot{\gamma})$, est donné sur la figure 16 , après ajustement de $(15)$ à des mesures sur viscosimètres Couette à cylindres coaxiaux (Quemada et Dufaux, 1977).

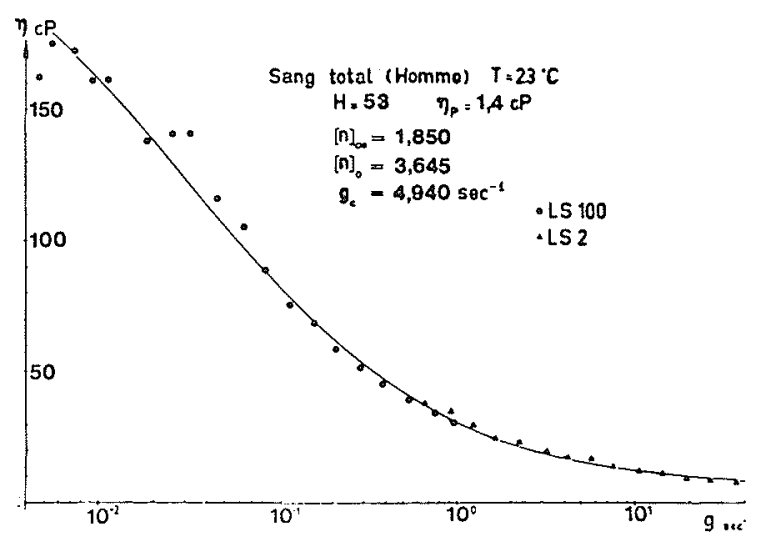

Figure 18 - Variations de la viscosité du sang total (Homme) en fonction de $\dot{\gamma}$ mesurées par viscosimétrie à cylindres co-axiaux (Contraves, Low Shear 100 et 2). Ajustement des paramètres de la relation (15).

(d'après Quemada et Dufaux, 1977).

\section{$B$ - Couche plasmatique et profil de vitesse}

La viscosité (15) varie surtout dans la région des $\dot{\gamma}$ encadrant $\dot{\gamma}_{c}$, c'est-à-dire dans la région voisine de l'axe (à très grands débits) ou voisine des parois (à très faibles débits) et cela, d'autant plus que la concentration est élevée. Il en résulte que :

1. Dans les tubes les plus larges $(20 a \leqslant R \leqslant 50 a)$, où, à forte concentration, l'épaisseur $\delta$ est négligeable $(1-\beta \ll 1)$, on observe des profils $v(r)$ quasi-paraboliques dans les deux limites des forts et faibles débits. Dans le domaine intermédiaire (débits moyens), le profil $v(r)$ est d'autant plus aplati que $c$ est élevée (Fig. 19). Cette région aplatie $\left(r \leqslant r_{b}\right)$ permet de considérer l'écoulement comme pseudo-diphasique, avec une phase centrale pratiquement solide entourée d'une phase plus fluide (cette augmentation de fluidité résultant essentiellement des valeurs élevées de $\dot{\gamma}$ près des parois).

2. Dans les plus petits tubes, un modèle diphasique est nécessaire, avec deux concentrations $c_{s}$ et $c_{w}$, cette dernière pouvant être nulle ou non. A forte concentration, on peut s'attendre à $c_{s}$ voisine de sa valeur maximum. La généralisation des relations (12) et (13) permet de déduire l'épaisseur de la couche plasmatique lorsque $c_{w}$ et le rapport $c_{t} / c_{o}$ sont connus. Le modèle en cours de développement conduit à une assez bonne vérification de l'hypothèse de Barbee-Cokelet (Cf. eq. (9)). La 


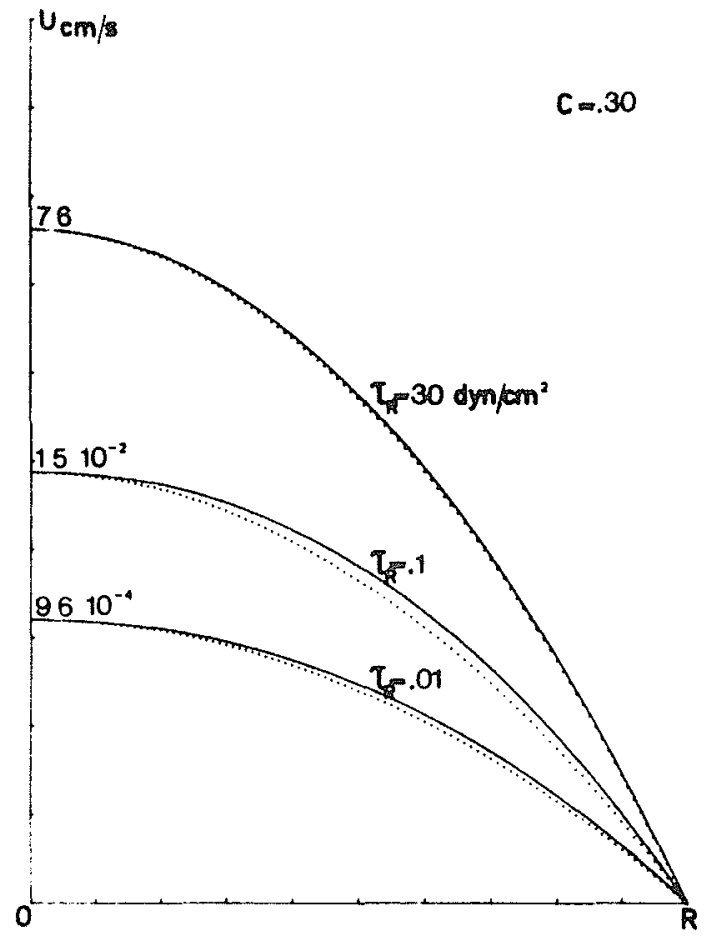

a)

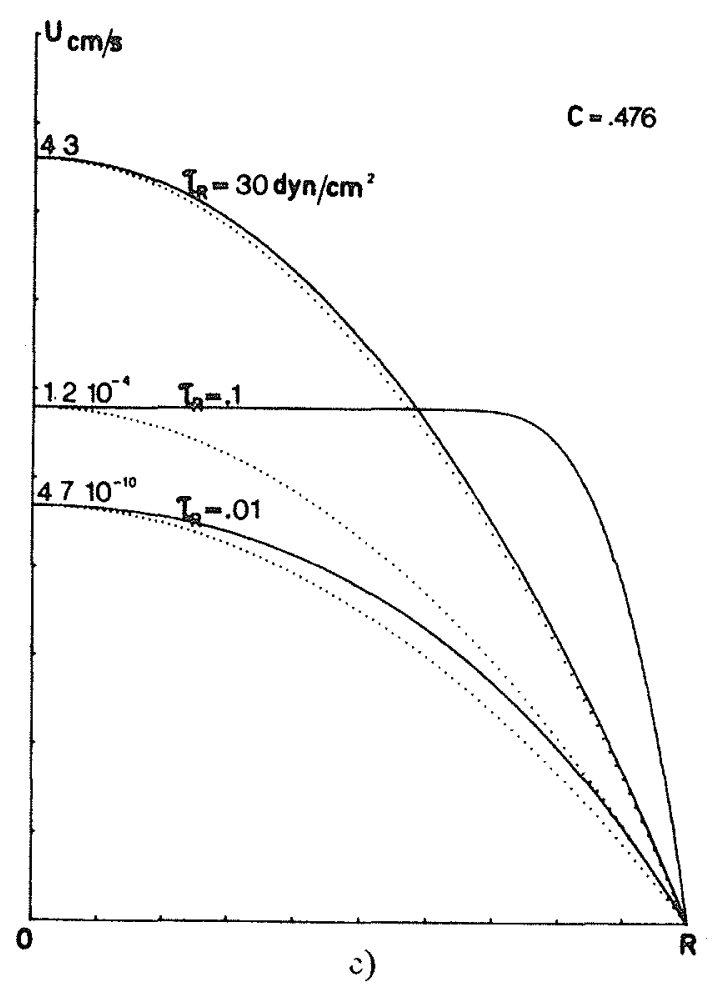

Figure 19 - Profils de vitesse d'un modèle monophasique nonnewtonien (Relation (15)) dans un tube de rayon $R O=1 \mathrm{~mm}$ pour trois concentrations $c=0,30 ; 0,45 ; 0,4761$ (la dernière étant très proche de la concentration maximum $c_{m}$ ) et pour différentes valeurs de $\tau_{R}$. Ces profils montrent un aplatissement pour $\tau_{R}=0,1$, d'autant plus important que $c_{s}$ est proche de $c_{m}$. (Le profil parabolique associé à la même vitesse maximum $U_{m}$ tracé en pointillé. Les échelles sont différentes pour les 3 profils, les valeurs $U_{m}$ ont étê portées sur la figure).

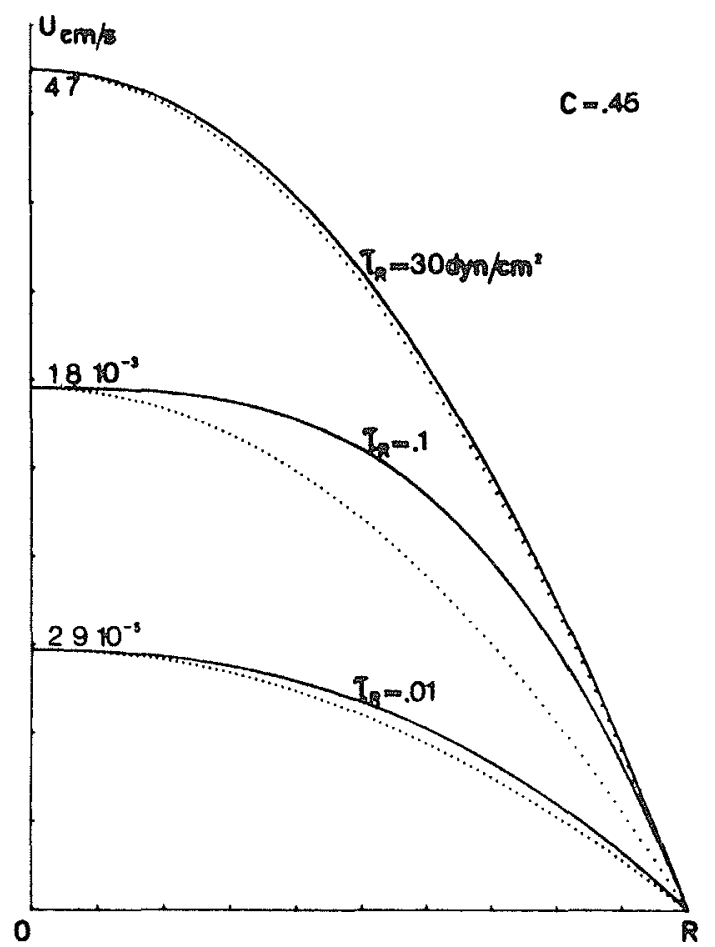

b)

figure 20 montre deux exemples (débits moyen et fort) de profils de vitesse calculés. Là encore, l'aplatissement n'est sensible que pour les débits moyens. Ces profils sont comparés aux profils obtenus pour deux fluides homogènes (écoulement monophasique)

(i) ayant la concentration moyenne du tube $c_{t}=C T$ (et donnant la même viscosité apparente que l'écoulement diphasique)

(ii) ayant la concentration du réservoir $c_{o}=C A$.

La comparaison de ces deux profils rend évidente la réduction de viscosité (de l'ordre de $50 \%$ ).

\section{Conclusion}

Les écoulements des milieux dispersés concentrés dans les conduits de faibles dimensions montrent une structure diphasique, avec une couche marginale appauvrie en particules. Cette couche marginale est d'une importance capitale. Non seulement elle permet un abaissement important de la viscosité apparente, mais aussi elle s'accompagne d'une augmentation du gradient de vitesse à la paroi. Pour la circulation sanguine, cela signifie une circulation plus aisée (évitant l'hypertension notamment), mais aussi, par l'accroissement du cisaillement à la paroi et l'augmentation des phénomènes de transport, une facilitation des échanges, sous conditions normales. Néanmoins, cela entraîne une grande fragilité vasculaire, qui, (sous conditions pathologiques) en font un terrain privilégié de lésions. L'approfondissement des problèmes posés et la compréhension des mécanismes 

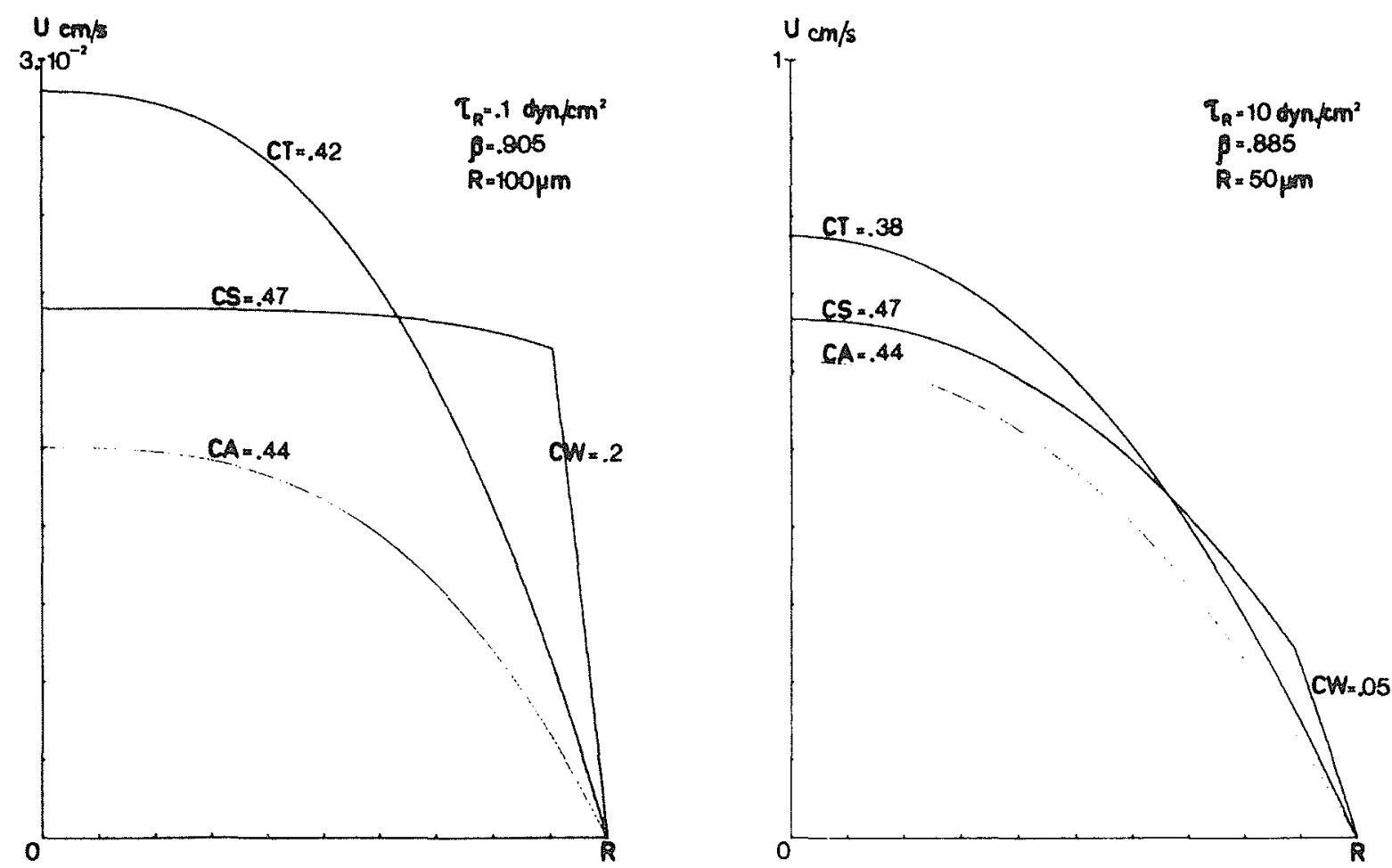

Figure 20 - Profils de vitesse d'un modèle diphasique (non-newtonien, relation (15), pour la région $(S)$ - newtonien pour la région (w)) et comparaison aux profils $u(r)$ monophasiques pour la concentration moyenne $C T$ (donnant la même viscosité apparente : hypothèse de Barbee Cokelet) et pour la même concentration débitante $C A$ (augmentation de la viscosité apparente).

physico-chimiques associés apparaît donc de grande importance, tant pour la physiopathologie que pour le développement des systèmes d'assistance extracorporelle (rein et poumon artificiels) ou des prothèses vasculaires. Enfin, le caractère très général des propriétés étudiées laisse espérer des retombées dans la plupart des domaines où l'on rencontre des écoulements de milieux dispersés concentrés.

\section{Références}

AROESTY J., GROSS J.F. (1970) - Convection and Diffusion in the Microcirculation; Microvasc. Res., 2, 247-267.

BARBEE J.H., COKELET G.R. (1971) - The Fahraeus effect ; Microvasc. Res., 3, 6-21.

BAYLISS L.E. (1952) - Rheology of blood and lymph in Deformation and flow in biological systems (ed. A. FREY. WYSSLING) pp. 355-418 - North Holland Publish $\mathrm{C}^{\circ}$ Amsterdam.

BROCHARD F. (1977) - Une bulle dégonflée : le globule rouge ; La Recherche, 75, 174-177.

BUGGLIARELLO G., KAPUR C., HSIAO G. (1965) - The profile viscosity and others characteristics of blood flow in a non-uniform shear field. Proc. Four Int. Cong. on Rheol., 4 (ed. COPLEY A.L.) pp. 351-370, Interscience, N.Y.

BURTON A.C. (1966) - Physiology and Biophysics of the circulation. Year Book Medic. Publ., Chicago.

CASSON N. (1959) - A flow equation for pigment-oil suspensions of printing ink type ; in Rheology of Disperse systems, (ed. MLLL C.C.) pp. 84-102, Pergamon, London.

CHARM S.E., KURLAND G.S., BROWN S.L. (1968) - The influence of radial distribution and marginal plasma layer on the flow of ted cells suspensions; Biorheol. 5, 15-43.
CHIEN S. (1970) - Shear dependance of effective cell volume as a determinant of blood viscosity ; Science 168, 977-979.

CHIEN S., USAMI S., DELLENBACK R.J., BRYANT C.A. (1971) - Comparative hemorheology. Hematological implications of species differences in blood viscosity ; Biorheol. 8, 35-57.

CHIEN S. (1972) in "Hemodilution": Theoretical Basis and Clinical Applications, p. $1-$ K. Messmer et H. SchmidSchönbein ed., - S. Karger, Munich.

COKELET G.R. (1972) - The rheology of human blood; in Biomechanics : its Foundations and Objectives (Y.C. FUNG, N. PERRONE and M. ANLIKER, ed.) Chap. 4, 63-103. Prentice Hall, Inc - New Jersey.

COKELET G.R. (1976) - Macroscopic rheology and tube flow of human blood in Micro-circulation Vol 1 (ed. J. GRAYSON and W. Zingg) 9-31, Plenum Press N.Y.

FAHRAEUS R. (1929) - The suspension stability of blood; Physiol. Rev., 9, 241-274.

FAHRAEUS R., LINDQVIST T. (1931) - The viscosity of the blood in narrow capillary tubes; Am. J. Physiol., 96, 562-568.

FITZ-GERALD J.M. (1969a) - Mechanics of red-cell motion through very narrow capillaries., Proc. Roy. Soc. London B 174, 193-227.

FITZ-GERALD J.M. (1969b) - Implications of a theory of ery throcyte motion in narrow capillaries ; $J$. Appl. Physiol. 27, 912-918.

FUNG Y.C. (1969) - Blood flow in the capillary bed. J. Biomechan., 2, 353-373.

GOLDSMITH H.L., MASON S.G. (1967) - The microrheology of Dispersions ; in Rheology (ed. F.R. EIRICH), Vol 5, 86-250. N.Y. Academic.

GREGERSEN M.I., BRYANT C.A., HAMMERLE W.E. USAMI S., CHIEN S. (1967) - Flow characteristics of human erythrocytes through polycarbonate sieves ; Science, $157,825-827$ 
HAYNES R.H., BURTON A.C. (1959) - Role of the nonnewtonian behaviour of blood in hemodynamics ; $A m . J$. Physiol., 197, 943-950.

HAYNES R.H. (1961) - The rheology of blood ; Trans. Soc Rheol., 5, 85-101.

HOCHMUTH R.M., MARPLE R.N., SUTERA S.P. (1970) Capillary blood flow : I Erythrocy te deformation in glass capillaries ; Microvasc. Res., 2, 409-419.

KARNIS A., GOLDSMITH H.L., MASON S.G. (1966) - The kinetics of flowing dispersions - I. Concentrated suspensions of rigid particles. J. Colloid and interf. Sci., 22, 531-553.

KUMIN K. (1949) - Inaugural dissertation, University of Bern. LEW H.S., FUNG Y.C. (1970) - Plug Effect of erythrocy tes in capillary blood vessels. Biophys. J., 10, 80-99.

LIGHTHILL M.J. (1968) - Pressure forcing of tightly fitting pellets along a fluid-filled elastic tubes ; $J$. Fluid. Mech., 34, 113-143.

LIGHTHILI M.J. (1969) - Motion in narrow capillaries from the stand-point of lubrication theory; in Ciba Symposium : "Circulatory and Mass Transport" (G. Westenholme, ed.) p. 85 - Little, Brown, Boston.

MAUDE A.D., WHITMORE R.L. (1956) - The wall effect and the viscosimetry of suspensions ; Br. J. Appl. Phys., 7,98-102.

MERRILL E.W., MARGETTS W.G., COKELET G.R., BRITTEN A., SALZMAN E.W., PENNEL R.B., MELIN M. (1965) Influence of plasma proteine on the rheology of human blood. Proc. Fourth. Int. On Rheol., 4 (ed. COPLEY A.L.) 601-611, Interscience, N.Y.

MIDDELMAN S. (1972) - Transport phenomena in the cardiovascular system (Chap. 2.91) Wiley-Interscience, N.Y.

OLIVER D.R., WARD S.G. (1953) - Relationship between relative viscosity and volume concentration of stable suspensions of spherical particles ; Nature, Lond. 171, 396-397.

PROTHERO J. et BURTON A.C. (1961) - The Physics of blood in capillaries: I The nature of the motion ; Biophys. $J .1$, 567.579 .
QUEMADA D. (1975) - Rhéologie des suspensions concentrées et du sang. Discussion d'une loi viscosité concentration déduite du principe d'énergie dissipée extrémale. Comptes Rendus 280 B, 793-797.

QUEMADA D. (1976) - Une nouvelle méthode de caractérisation du sang. Comptes Rendus 282 D, 1905-1909.

QUEMADA D. (1977) - Rheology of concentrated disperse system and minimum energy dissipation principle. I Viscosity-concentration relationship. Rheol. Acta, 16, 82-94.

QUEMADA D. (1978) - Rheology of concentrated disperse systems. II. A model for non newtonian shear viscosity in steady flows. Rheologica acta (accepté).

QUEMADA D., DUFAUX J. (1977) - Vers une caractérisation rhéologique du sang. Congrès de Pathologie Vasculaire, Paris.

RAND R.P. (1964) - Mechanical properties of the red cell membrane : II Viscosity breakdown. Biophys. J., 4, 303-316.

SKALAK R. et BRANEMARK P.I. (1969) - Deformation of red cells in capillaries. Science 14, 717-742.

SKALAK R., CHEN P.M., CHIEN S. (1972) - Effect of hematocrit and rouleaux on apparent viscosity in capillaries. Biorheol., 9, 67-82.

SESHADRI V., SUTERA S.P. (1970) - Apparent viscosity of coarse-concentrated suspensions in tube flow. Trans. Soc. Rheol. 14, 351-373.

SUTERA S.P., SESHADRI V., CROCE P.A., HOCHMUTH R.M. (1970) - Capillary blood Flow.II.Deformable Model Cells in tube flow. Microvascular Research, 2, 420-433.

THOMAS H.W. (1961) - The wall effect in capillary instruments : an improved analysis suitable for application to blood and other particulate suspensions. Biorheol, 1, 41-56.

VAND V. (1948) - Viscosity of solutions and suspensions. $J$. Phys. Colloid. Chem., 52, 277-299; 52, 300-321.

WHITMORE R.L. (1968) - Rheology of the circulation. Pergamon Press (Oxford)

\section{Discussion}

\section{Président : M.Y. JAFFRIN}

Le Professeur LEMAIRE félicite M. QUEMADA pour la clarté de son exposé et insiste sur tout l'intérêt qu'il présente pour les physiologistes et les biologistes. Il attire l'attention sur l'extrême difficulté des études relatives à la microcirculation et à la circulation dans les capillaires: "Nous savons que ces capillaires sont fenestrés, que leurs parois ne sont pas continues. Pourquoi? Pourquoi toutes ces forces mécaniques qui ont été décrites ne jouent pas? Pourquoi le globule rouge comprimé dans un petit vaisseau quand il passe devant une fenêtre reste dans ce vaisseau ?"

M. QUEMADA confirme qu'en dépit de très nombreuses études, ce problème - comme bien d'autres - est loin d'être élucidé.

Il évoque notamment une série d'essais sur la microcirculation rénale à l'aide de petites sphères. Leur distribution ne se révèle pas du tout conforme à ce que l'on pourrait imaginer du comportement de petites sphères dans des tuyaux.

Autant, précise-t-il, de sérieux progrès ont été enregistrés concernant l'analyse des écoulements pulsatifs dans les gros vaisseaux, autant on est encore à essayer de comprendre les grands traits de la microcirculation.

M. COMOLET observe qu'en situation normale un capillaire sur trois environ est le siège d'un écoulement sanguin; dans les deux autres le sang ne circule pas. Il demande si le sang au repos participe aux échanges transcapillaires, comme on l'admet pour le sang en mouvement - et, si oui, dans quelle mesure - ?
M. QUEMADA rappelle à ce sujet l'effet de "court-circuit" de l'anastomose veinulo-capillaire, qui évite les coups de bélier dans la circulation amont lors des arrêts intervenant au niveau de la microcirculation, arrêts provoqués par des petits sphincters au fonctionnement encore un peu mystérieux.

Il pense que, concernant les échanges, la situation d'écoulement est plus favorable car en situation de non-écoulement il ne faudrait compter que sur des transferts par diffusion. Or si les coefficients de diffusion de la plupart des produits véhiculés (gaz ou oxygène notamment) sont de l'ordre de $10^{-5} \mathrm{~cm} / \mathrm{sec}$, celui des protéines est considérablement plus faible et il n'y aurait plus aucun échange possible.

On est tenté d'en conclure qu'il est essentiel que le sang circule pour que les échanges aient lieu. Mais dire cela est une profession de foi : on ne l'a pas établi clairement.

M. KOPP remarque que la microcirculation semble de type aléatoire et que cela intervient probablement dans l'échange veineux.

M. QUEMADA observe qu'il faut distinguer à cet égard les petits vaisseaux, de la taille au-dessus des capillaires, où il y a peu d'échanges, et les capillaires eux-mêmes. Selon les observations qui ont pu être faites, le globule rouge qui se trouve dans un capillaire n'y a peut être pas un mouvement continu: il s'arrête, il repart mais ce ne sont pas vraiment des conditions aléatoires. 